\title{
A Model of Hereditary Sensory and Autonomic Neuropathy Type 1 Reveals a Role of Glycosphingolipids in Neuronal Polarity
}

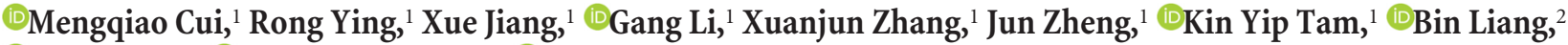 \\ ๑Anbing Shi, ${ }^{3}$ @Verena Göbel, ${ }^{4}$ and ${ }^{\circledR}$ Hongjie Zhang ${ }^{1,5}$ \\ ${ }^{1}$ Faculty of Health Sciences, University of Macau, Taipa, Macau SAR 999078, China, ${ }^{2}$ Key Laboratory of Animal Models and Human Disease Mechanisms, \\ Kunming Institute of Zoology, Chinese Academy of Sciences, Kunming, Yunnan 650223, China, ${ }^{3}$ Department of Biochemistry and Molecular Biology, \\ School of Basic Medicine, Tongji Medical College, Huazhong University of Science and Technology, Wuhan, Hubei 430030, China, ${ }^{4}$ Mucosal Immunology \\ and Biology Research Center, Department of Pediatrics, Massachusetts General Hospital and Harvard Medical School, Boston, Massachusetts 02114, and \\ ${ }^{5}$ Center of Reproduction, Development and Ageing, University of Macau, Taipa, Macau SAR 999078, China
}

Hereditary sensory and autonomic neuropathy Type 1 (HSAN1) is a rare autosomal dominantly inherited neuropathy, clinically characterized by a loss of distal peripheral sensory and motoneuronal function. Mutations in subunits of serine palmitoyltransferase (SPT) have been linked to the majority of HSAN1 cases. SPTs catalyze the condensation of L-serine with palmitoyl-CoA, the first committed and rate-limiting step in de novo sphingolipid biosynthesis. Despite extensive investigation, the molecular pathogenesis of HSAN1 remains controversial. Here, we established a Caenorhabditis elegans (C. elegans) model of HSAN1 by generating a sptl-1(c363g) mutation, encoding SPTL-1(C121W) and equivalent to human SPTLC1 ${ }^{\mathrm{C} 133 \mathrm{~W}}$, at the $C$. elegans genomic locus through CRISPR. The sptl-1(c363g) homozygous mutants exhibited the same larval lethality and epithelial polarity defect as observed in sptl-1(RNAi) animals, suggesting a loss-of-function effect of the SPTL-1(C121W) mutation. sptl-1(c363g)/+ heterozygous mutants displayed sensory dysfunction with concomitant neuronal morphology and axon-dendrite polarity defects, demonstrating that the $C$. elegans model recapitulates characteristics of the human disease. sptl-1(c363g)-derived neuronal defects were copied in animals with defective sphingolipid biosynthetic enzymes downstream of SPTL-1, including ceramide glucosyltransferases, suggesting that SPTLC1 ${ }^{\text {C133W }}$ contributes to the HSAN1 pathogenesis by limiting the production of complex sphingolipids, including glucosylceramide. Overexpression of SPTL-1(C121W) led to similar epithelial and neuronal defects and to reduced levels of complex sphingolipids, specifically glucosylceramide, consistent with a dominant-negative effect of SPTL-1(C121W) that is mediated by loss of this downstream product. Genetic interactions between SPTL$1(\mathrm{C} 121 \mathrm{~W})$ and components of directional trafficking in neurons suggest that the neuronal polarity phenotype could be caused by glycosphingolipid-dependent defects in polarized vesicular trafficking.

Key words: axonal transport; glucosylceramide; HSAN1; neuronal polarity; sphingolipidomics; sphingolipids

\section{Significance Statement}

The symptoms of inherited metabolic diseases are often attributed to the accumulation of toxic intermediates or byproducts, no matter whether the disease-causing enzyme participates in a biosynthetic or a degradation pathway. By showing that the phenotypes observed in a C. elegans model of HSAN1 disease could be caused by loss of a downstream product (glucosylceramide) rather than the accumulation of a toxic byproduct, our work provides new insights into the origins of the symptoms of inherited metabolic diseases while expanding the repertoire of sphingolipid functions, specifically, of glucosylceramides. These findings not only have their most immediate relevance for neuroprotective treatments for HSAN1, they may also have implications for a much broader range of neurologic conditions.

\section{Introduction}

Hereditary sensory and autonomic neuropathy Type 1 (HSAN1) is a rare progressive neurological disorder, clinically character- ized by distal peripheral sensory loss and disturbances in motoneuronal function, genetically identified to be a heterogeneous condition associated with mutations in several genes (Dawkins et 
al., 2001; Rotthier et al., 2012). Among these genes, mutations in the first (SPTLC1) and second (SPTLC2) subunits of the enzyme serine palmitoyltransferase have been linked to the majority of HSAN1 cases. SPTLC1 ${ }^{\mathrm{C} 133 \mathrm{~W}}$ is the most frequently identified mutation in patients (Bejaoui et al., 2001; Rotthier et al., 2009).

Despite extensive investigation, the molecular pathogenesis of HSAN1 remains controversial. Initially, it was believed to be caused by a loss of SPT function, which is supported by evidence for reduced SPT activity and decreased total sphingolipid (SL) levels in the presence of a mutant SPTLC1 allele (Bejaoui et al., 2002; Gable et al., 2002). However, this hypothesis is challenged by reports of unchanged or even increased SL quantities in symptomatic HSAN1 patients and SPTLC1 ${ }^{\mathrm{C} 133 \mathrm{~W}}$ transgenic mice (Dedov et al., 2004; McCampbell et al., 2005). Recently, arguments for a "gain-of-function" scenario have been advanced, based on the identification of a common feature of almost all examined HSAN1-causing SPT mutations: a shift in SPT substrate specificity for L-alanine and L-glycine over L-serine to form deoxysphingoid bases (DSBs), with the accumulation of deoxy-SLs thought to be the toxic cause of the nervous system phenotype (Eichler et al., 2009; Penno et al., 2010; Rotthier et al., 2010, 2011; Garofalo et al., 2011).

However, elevated deoxy-SL levels have also been reported in conditions with intact SPT activity (Zitomer et al., 2009; Bertea et al., 2010). Moreover, the SPT activity is more severely reduced in SPTLC1 ${ }^{\mathrm{C} 133 \mathrm{~W}}$ transgenic mice carrying two wild type (WT) alleles than in heterozygous SPTLC1 KO mice (Eichler et al., 2009). Therefore, it is still possible that the observed phenotypic changes in HSAN1 are due to a dominant-negative lossof-function scenario.

We previously demonstrated that loss of C. elegans SPT, as well as the loss of 14 of its downstream SL biosynthetic enzymes, resulted in the same specific epithelial polarity phenotype (Zhang et al., 2011), demonstrating that the loss of an SPT product (in this case glucosylceramide) caused this phenotype. Moreover, our subsequent analysis suggested that the polarity phenotype resulted from a defect in apical trafficking (Zhang et al., 2011, 2012). Such a defect could also be a pathogenetic mechanism for the HSAN1-specific long fiber neuropathy, since polarized transport of membrane components to the tip of neurons is critical for function (Bentley and Banker, 2016). Consistent with this idea, a Drosophila model of HSAN1 has implicated perturbed ER to Golgi trafficking in the pathogenesis of nociceptive defects in HSAN1 (Oswald et al., 2015). We therefore hypothesized that the HSAN1 neuropathy might be caused by similar cellular defects as those shown to cause the C. elegans SL loss-of-function epithelial phenotype, and took advantage of this opportunity to use this model organism for the genetic and cell biological analysis of HSAN1.

Here, we established a C. elegans model of HSAN1 by introducing the disease-causing mutation SPTLC1 ${ }^{\mathrm{C} 133 \mathrm{~W}}$ at the $C$. elegans genomic locus, designated as sptl-1(c363g) according to its DNA sequence, thereby generating strains that recreate the exact genetic scenario of human HSAN1. We also generated

2016-00066-FHS and 2017-00082-FHS. We thank the C. elegans Genetic Center, funded by National Institutes of Health Office of Research Infrastructure Programs (P40 0D010440), and National Bioresource Project (Japan) for strains; Hong Zhang for assistance with CRISPR-based gene editing; and Garry G. Wong and members of the H.Z. and Wong laboratories for reagents and discussion.

The authors declare no competing financial interests.

Correspondence should be addressed to Hongjie Zhang at hizhang@umac.mo or Verena Göbel at vgobel@mgh.harvard.edu.

https://doi.org/10.1523/JNEUROSCI.2541-18.2019

Copyright $\odot 2019$ the authors
SPTLC1 ${ }^{\mathrm{C} 133 \mathrm{~W}}$-overexpressing transgenic lines, referred to as SPTL-1(C121W) based on the C. elegans protein sequence. Taking advantage of the complementarity of the two models, we set out to elucidate the molecular pathogenesis of HSAN1. The models we generated provide the basis for future in vivo genetic and drug screens to seek strategies and compounds for the treatment of HSAN1-derived neurodegeneration.

\section{Materials and Methods}

Strains and genetics. C. elegans strains were grown, maintained, and crossed using standard techniques described previously (Brenner, 1974). Most of the strain culture and experiments were performed at $20^{\circ} \mathrm{C}-22^{\circ} \mathrm{C}$ unless otherwise specified. The Bristol N2 strain (RRID:WB-STRAIN: N2_(ancestral)) was used as WT reference. Strains used are shown in Table 1.

CRISPR-based sptl-1(c363g) mutant generation. For generating a DNA double-strand break at sptl-1 genomic locus, a 20 bp sptl-1 specific guide sequence beginning with a $\mathrm{G}$ to optimize U6-driven transcription and ending with an NGG PAM site, was designed using gRNA finder. The sequence of $20 \mathrm{bp}$ gRNA is GTAGGATCGTGCGGGCCACG. The $20 \mathrm{bp}$ gRNA was cloned into pPD162 Peft-3::Cas9;PU6::empty sgRNA vector (a gift from Bob Goldstein, Addgene plasmid \# 47549). To make SPTL$1(\mathrm{C} 121 \mathrm{~W})$ mutation, an exogenous donor oligonucleotide was designed and synthesized to bear the sptl-1(c363g) mutation, encompassing $50 \mathrm{bp}$ of flanking homology on either side, and additional silent mutations to ablate the gRNA cleavage site and introduce a restriction site for ease of screening by restriction digest after single-worm PCR. To make animals that have successfully experienced homologous recombination event easily discernable, $d p y-10$ (cn64) mutation, which confers a dominant rolling phenotype in the $\mathrm{F} 1 \mathrm{mut} /+$ animals, was used as a marker (Arribere et al., 2014). The vector pPD162 containing a sptl-1 gRNA and a donor oligonucleotide to create sptl-1(c363g) point mutation were coinjected with the vector containing a $d p y-10$ gRNA (Addgene, plasmid 59933) and a donor oligonucleotide to create the $d p y$-10(cn64) mutation. F1 progeny were screened for the Rol phenotype. Roller animals were singled, and after laying eggs, were screened by single-worm PCR and restriction digest for characterization of the sptl-1(c363g) mutation. Nonroller F2 progeny of appropriate F1 animals were singled and screened for homozygosity of the sptl-1(c363g) mutation. The specificity of mutations was confirmed by sequencing. To exclude the presence of other DNA changes at and outside sptl-1, animals carrying the germline sptl-1(c363g) mutation were multiple times backcrossed and the full genomic locus, including $1.3 \mathrm{~kb}$ upstream and $1 \mathrm{~kb}$ downstream flanking sequence, was also sequenced.

RNAi knockdown. To assess epithelial defects and lethality, sptl-1 RNAi was performed as previously described (Zhang et al., 2011). To evaluate the phenotypes in L1-stage DD neurons, L4 stage CZ333 (unc-25p:: snb-1::gfp, RRID:WB-STRAIN:CZ333) or cgt-1(ok1045) (RRID:WBSTRAIN:VC693); CZ333 animals were transferred to RNAi plates (containing $50 \mu \mathrm{g} / \mathrm{ml}$ carbenicillin and $2 \mathrm{~mm}$ isopropylthiogalactoside), seeded with sptl-1 and cgt-3 RNAi bacteria, respectively, and allowed to produce progeny at room temperature. The progeny determined to be at the L1 stage $(\sim 15-16 \mathrm{~h}$ after egg-laying; mutant/RNAi animals grow slower) was examined under a confocal microscope. To evaluate the phenotypes in L4-stage ASI and RIA neurons, eggs were isolated from gravid hermaphrodites by a standard bleaching protocol (Zhang et al., 2015) and allowed to hatch directly on the RNAi plates seeded with sptl-1 and cgt-3 RNAi bacteria. The worms were scored for phenotypes when they had developed into L4 larvae. For RNAi controls, worms were fed with RNAi bacteria containing the empty vector L4440. Bacterial clones were picked from the Ahringer genome-wide RNAi feeding library (J. Ahringer, Wellcome Trust/Cancer Research UK Gurdon Institute, Cambridge, UK).

Molecular cloning and site-directed mutagenesis. Translational fusion constructs were generated in the pPD95.75 vector (a gift from Andrew Fire, Addgene, plasmid \#1494). To express C. elegans SPTL-1 and CGT-3, first-strand cDNAs were generated from total RNA using random hexamer primers by Superscript II Reverse Transcriptase, as described in the 
Table 1. C. elegans strains used in this study

\begin{tabular}{|c|c|c|}
\hline Strain name & Genotype & Source \\
\hline CZ333 & juls1 [unc-25p::snb-1::GFP + lin-15(+)]/V & CGC \\
\hline CX3572 & kyls105[str-3p::snb-1::GFP + lin-15(+)] & CGC \\
\hline CZ1200 & juls76[unc-25p::GFP + lin-15(+)]II & CGC \\
\hline IK721 & njls9 [glr-3p::snb-1::Venus + ofm-1::GFP]/V & CGC \\
\hline VJ402 & fgEx13[erm-1p::erm-1::GFP, rol-6p::rol-6(su1006)] & V.G. laboratory \\
\hline VC693 & cgt-1(ok1045) V & $\mathrm{CGC}$ \\
\hline RB1498 & hyl-2(ok1766)X & CGC \\
\hline DR1 & unc-101(m1)I & CGC \\
\hline Eu828 & dhc-1(or195)I & CGC \\
\hline FF41 & unc-116(e2310) III & CGC \\
\hline RB662 & $a p b-3(0 k 429) I$ & CGC \\
\hline CB1111 & cat-1(e1111)X & CGC \\
\hline CB1370 & daf-2(e1370) III & CGC \\
\hline CX2065 & odr-1(n1936)X & CGC \\
\hline HJZOO1 & sptl-1(c363g)/+II & This study \\
\hline HJZOO2 & sptl-1(c363g)/+ Il;fgEx13 [erm-1p:: erm-1::GFP, rol-6p::rol-6(su1006)] & This study \\
\hline HJZOO3 & sptl-1(c363g)/+II;juls76[unc-25p::GFP + lin-15(+)]/I & This study \\
\hline HJZOO4 & sptl-1(c363g)/+ II; juls1 [unc-25p::snb-1::GFP + lin-15(+)]IV & This study \\
\hline HJZOO5 & sptl-1(c363g)/+ ll;kyls105[str-3p::snb-1::GFP + lin-15(+)] & This study \\
\hline HJZOO6 & sptl-1(c363g)/+ II;njls9[glr-3p:::snb-1::Venus + ofm-1::GFP]/V & This study \\
\hline HJZOO7 & juls1 [unc-25p::snb-1::GFP + lin-15(+)]IV; cgt-1(ok1045) V & This study \\
\hline HJZOO8 & cgt-1(ok1045) V; kyls105 [str-3p::snb-1::GFP + lin-15(+)] & This study \\
\hline HJZOO9 & juls76[unc-25p::GFP + lin-15(+)]Il;cgt-1(ok1045) V & This study \\
\hline HJZO10 & njls9 [glr-3p:::snb-1::Venus + ofm-1::GFP]/V; cgt-1(ok1045) V & This study \\
\hline HJZO11 & juls1 [unc-25p::snb-1::GFP + lin-15(+)]IV; hyl-2(ok1766)X & This study \\
\hline HJZO12 & hyl-2(ok1766) X; kyls105 [str-3p::snb-1::GFP + lin-15(+)] & This study \\
\hline HJZO13 & njls9 [glr-3p::snb-1::Venus + ofm-1::GFP]IV; hyl-2(ok1766)X & This study \\
\hline HJZO14 & mauEx01 [sptl-1p:::sptl-1::GFP] & This study \\
\hline HJZO15 & mauEx02 [sptl-1p::SPTL-1(C121W):::GFP] & This study \\
\hline HJZO16 & mauEx03 [rgef-1p:::sptl-1::mCherry] & This study \\
\hline HJZO17 & mauEx04[rgef-1p:::SPTL-1(C121W)::mCherry] & This study \\
\hline HJZO18 & mauEx05[sptl-1p::cgt-3:::mCherry] & This study \\
\hline HJZO19 & mauEx06 [rgef-1p:::cgt-3::mCherry] & This study \\
\hline HJZO2O & mauls01 [spt-1p::sptl-1::GFP] & This study \\
\hline HJZO21 & mauls02[sptl-1p::SPTL-1(C121W):::GFP] & This study \\
\hline HJZO22 & sptl-1(c363g) II; fgEx13 [erm-1p::erm-1::gfp, rol-6p::rol-6(su1006)]; mauEx01 [sptl-1p::sptl-1::GFP] & This study \\
\hline HJZO23 & sptl-1(c363g)/+ Il; fgEx13 [erm-1p::erm-1::GFP, rol-6p::rol-6(su1006)]; mauEx02 [sptl-1p::SPTL-1(C121W)::GFP] & This study \\
\hline HJZO24 & sptl-1(c363g) II; fgEx13 [erm-1p::erm-1::gfp, rol-6p::rol-6(su1006)]; mauEx03 [rgef-1p::sptl-1::mCherry] & This study \\
\hline HJZO25 & sptl-1(c363g)/+ Il; fgEx13 [erm-1p:::erm-1::GFP, rol-6p::rol-6(su1006)]; mauEx04[rgef-1p::SPTL-1(C121W)::mCherry] & This study \\
\hline HJZO26 & sptl-1(c363g) II; fgEx13 [erm-1p::erm-1::GFP, rol-6p::rol-6(su1006)]; mauEx05 [sptl-1p:::cgt-3::mCherry] & This study \\
\hline HJZO27 & sptl-1(c363g)/+ Il;fgEx13 [erm-1p::erm-1::GFP, rol-6p::rol-6(su1006)]; mauEx06[rgef-1p::cgt-3::mCherry] & This study \\
\hline HJZO28 & fgEx13 [erm-1p::erm-1::GFP, rol-6p:::rol-6(su1006)]; mauEx01 [sptl-1p:::sptl-1::GFP] & This study \\
\hline HJZO29 & fgEx13[erm-1p::erm-1::GFP, rol-6p::rol-6(su1006)]; mauEx02 [sptl-1p::SPTL-1(C121W):::GFP] & This study \\
\hline HJZO3O & sptl-1(c363g)/+ Il; fgEx13 [erm-1p::erm-1::GFP, rol-6p::rol-6(su1006)]; mauls02 [sptl-1p::SPTL-1(C121W)::GFP] & This study \\
\hline HJZO31 & juls76[unc-25p::GFP + lin-15(+)]ll; mauEx03 [rgef-1p:::sptl-1::mCherry] & This study \\
\hline HJZO32 & juls76[unc-25p::GFP + lin-15(+)]ll; mauEx04 [rgef-1p::SPTL-1(C121W)::mCherry] & This study \\
\hline HJZO33 & njls9 [glr-3p::snb-1::Venus + ofm-1::GFP]/V; mauEx03 [rgef-1p:::sptl-1::mCherry] & This study \\
\hline HJZO34 & njls9 [g/r-3p:::snb-1::Venus + ofm-1::GFP] IV; mauEx04[rgef-1p::SPTL-1(C121W)::mCherry] & This study \\
\hline HJZO35 & $a p b-3($ ok429) l; njls9 [glr-3p::snb-1::Venus + ofm-1::GFP] $/ V$ & This study \\
\hline HJZO36 & unc-101(m1) l; njls9[glr-3p::snb-1::Venus + ofm-1::GFP]/V & This study \\
\hline HJZO37 & apb-3(ok429) l; sptl-1(c363g)/+ II; njls9 [glr-3p::snb-1::Venus + ofm-1::GFP]IV & This study \\
\hline HJZO38 & unc-101(m1) l; sptl-1(c363g)/+ II; njls9[glr-3p:::snb-1::Venus + ofm-1::GFP]IV & This study \\
\hline HJZO39 & dhc-1(or195) l; juls1 [unc-25p::snb-1::GFP + lin-15(+)]IV & This study \\
\hline HJZO4O & unc-116(e2310) III; juls1 [unc-25p::snb-1::GFP + lin-15(+)]IV & This study \\
\hline HJZO41 & dhc-1(or195) l; sptl-1(c363g)/+ II; juls1 [unc-25p::snb-1::GFP + lin-15(+)]/V & This study \\
\hline HJZO42 & sptl-1(c363g)/+ II; unc-116(e2310) III; juls1 [unc-25p:::snb-1::GFP + lin-15(+)]/V & This study \\
\hline HJZO43 & dhc-1(or195) I; sptl-1(c363g)/+ II; unc-116(e2310) III; juls1 [unc-25p::snb-1::GFP + lin-15(+)]IV & This study \\
\hline HJZO44 & mauEx03 [rgef-1p::sptl-1::mCherry]; unc-116(e2310) III & This study \\
\hline
\end{tabular}

manufacturer's manual (Thermo Fisher Scientific). Using first-strand cDNA as templates, double-stranded sptl-1 and cgt-3 cDNAs were amplified by PCR, using sptl-1- and cgt-3-specific primers containing the restriction sites BamH I and KpnI, and inserted in frame with the GFP coding sequence into the plasmid vector pPD95.75 to generate sptl-1::gfp and $c g t-3:: g f p$ plasmids by basic subcloning procedures. SPTL-1(C121W) mutations were generated through site-directed mutagenesis (QuikChange Site-Directed Mutagenesis Kit, Agilent Technologies) after the sptl-1::gfp plasmid was obtained. Briefly, a pair of primers containing the sptl-1 (c363g) mutation were annealed to the corresponding sequences on opposite strands of the sptl-1::gfp template plasmid and extended in opposite directions. After 18 amplification cycles, PCR products were di- 
gested by DpnI to degrade the parental methylated plasmid and subsequently transformed into XL10-Gold ultracompetent cells. Plasmid DNAs were prepared from transformed bacteria and sequenced to verify the desired mutations.

To drive the expression of SPTL-1 or SPTL-1(C121W) in different tissues, DNA fragments of tissue-specific promoters were amplified from genomic DNA or plasmids by PCR (Expand Long Template or Expand High Fidelity PCR Systems, Roche Molecular Biochemicals) and joined with the cDNA-GFP coding sequence and the 3 '-UTR by the stitching method (Hobert, 2002). DNAs were prepared from multiple independent isolates, verified by restriction digest and sequencing, and a mixture was used for germline transformation of animals by microinjection (Mello et al., 1991). Constructs were normally injected at 50-100 ng/ul, along with appropriate selection markers. At least three independent transgenic lines were generated for each construct. Oligonucleotide sequences used are available upon request.

Epithelial and neuronal phenotype analysis. Epithelial and neuronal markers were introduced into the sptl-1(c363g)/+ and other mutants or transgenic animals by standard cross procedures. Genotypes of different mutants were confirmed by single-worm PCR. The F1 progeny from heterozygous parents was scored for the presence of epithelial or neuronal phenotypes. The epithelial polarity defects were scored under the dissecting microscope, whereas the neuronal phenotypes were scored under the confocal microscope. Dorsal SNB-1::GFP mislocalization in DD neurons was evaluated under the confocal microscope in $\mathrm{F} 1$ progeny from sptl-1(c363g)/+ parents, determined to be at the L1 stage. The penetrance of the phenotype in the F1 progeny of sptl-1(c363g)/+ parents was calculated based on the results from a mixed population, which theoretically contained 25\% WT, 50\% heterozygous, and 25\% homozygous animals. Axon outgrowth phenotype in DD neurons and SNB-1:: GFP mislocalization in RIA and ASI neurons were scored in L4 stage animals. Quantification for these neuronal phenotypes was based on the evaluation of a mixed F1 population from $s p t l-1(c 363 g) /+$ parents that contained one-third of WT and two-thirds of heterozygous animals (homozygous animals die as L1s). The presence of one-third of WT animals is expected to reduce the phenotype penetrance in this group.

Brood size assay. L4 larvae from heterozygous parents, determined to be sptl-1(c363g)/+ by the presence of the epithelial polarity defect and larval lethality in their $\mathrm{F} 1$ progeny, were singled onto OP50-seeded nematode growth medium (NGM) plates and subsequently transferred onto a new OP50 plate every $24 \mathrm{~h}$, and the number of newly hatched larvae was counted. This was repeated for $4 \mathrm{~d}$ until the parent worms stopped laying eggs. Each day, the progeny production was recorded and was compared with the WT controls. Thirty worms were observed at a time, and the experiments were repeated 3 times.

Life span assay. To analyze the life span of sptl-1(c363g)/+ mutants, we singled out 120-150 L4 larvae and determined their genotype by examining whether they produced homozygous mutant progeny. Then we transferred the animals whose genotype was identified to be sptl$1(c 363 g) /+$ to plates with OP50 for life span assays. Worms were cultured at $20^{\circ} \mathrm{C}$ and transferred every day to a new plate until they stopped laying eggs. Dead and alive worms were monitored twice a day until death. Worms were considered dead when they no longer responded to gentle touch with a platinum wire. Worms that crawled off the plates during the assay were replaced from a backup group of the same age. The experiments were repeated 3 times. Life span was defined as the time from the day of L4-adult transition until the day of death. Life span curves were analyzed using the Mantel-Cox test $(p<0.001)$.

Locomotion behavior assays. One-day-old young adult progeny from sptl-1(c363g)/+ heterozygous parents were placed onto a food-free plate. Worms were allowed to acclimate for 1 min before the start of the assay. Spontaneous reversal behavior was defined as any perceptible backward movement of the entire animal, counted by direct observation of free moving animals under a dissecting microscope over a 3 min period. Omega turns were visually scored as a sharp turn occurring immediately after a reversal, with the head nearly touching the tail or a reorientation of the worm body $>135^{\circ}$ from the reversal starting point. For each dwelling behavior experiment, 100 animals were picked to NGM plates seeded with equal amounts of OP50 in similar bacterial lawn sizes. After $30 \mathrm{~min}$, the worms that stayed on OP50 bacteria were counted. The genotypes of the animals were determined by examining their progeny after completion of the locomotion behavior assay, and only heterozygous animals were included for quantification.

Memory formation and food choice assay. A total of 80-100 adult F1 progeny from WT and heterozygous mutant parents were picked onto NGM plates with OP50, and allowed to lay eggs for $1.5 \mathrm{~h}$ before being killed. After $3 \mathrm{~d}$, the worms were washed with M9 buffer, rinsed three times, transferred to a PA14 (RRID:WB-STRAIN:PA14)-seeded plate, and trained for $12 \mathrm{~h}$. Adult worms were then washed from PA14 plates with M9 buffer, rinsed twice, and 100-200 animals were placed in the middle of the assay plate, equidistant from the bacterial lawns. The number of worms present on either bacterial lawn was counted, and their genotypes were determined by single-worm PCR followed by restriction digestion, and only heterozygotes were included for quantification. Memory formation and learning ability were evaluated through calculating Choice Index $(\mathrm{CI})=\left[\mathrm{n}_{\mathrm{PA} 14}-\mathrm{n}_{\mathrm{OP} 50}\right] /\left[\mathrm{n}_{\mathrm{PA} 14}+\mathrm{n}_{\mathrm{OP} 50}\right]$ and Learning Index $(\mathrm{LI})=\mathrm{CI}_{\text {naive }}-\mathrm{CI}_{\text {trained }}$. The higher LI, the better memory and learning ability.

Odorant chemotaxis and adaptation assay. Chemotaxis between $95 \%$ ethanol and $1 \%$ benzaldehyde was performed at $20^{\circ} \mathrm{C} ; 2 \mu \mathrm{l}$ each of ethanol and benzaldehyde was dropped onto an unseeded NGM plate. The two spots were 1.5 inches apart from each other and air-dried for $10 \mathrm{~min}$; $1 \mu \mathrm{l}$ of $1 \mathrm{~m} \mathrm{NaN} 3$ was applied to these two spots beforehand. The chemotaxis assay was performed by placing 100-200 progeny from heterozygous parents at a point on the plate equidistant from the two spots. For the adaptation assays, well-fed young adult animals (harvested $72 \mathrm{~h}$ after egg-laying) were preexposed to $100 \%$ benzaldehyde ( $2 \mu \mathrm{l}$ on the lid) for $1 \mathrm{~h}$ in the absence of food and then transferred to test plates as described previously (Nuttley et al., 2002). Worms were counted as having made their choice only if they were immobilized within $1 \mathrm{~cm}$ of the test spots at the time points when observations were made. Genotypes of the animals that had made their choice were determined by single-worm PCR followed by restriction digestion and only heterozygotes were included for quantification. CI was calculated as follows: $\mathrm{CI}=\left[\mathrm{n}_{\text {Benzaldehyde }}-\mathrm{n}_{\text {Ethanol }}\right] /\left[\mathrm{n}_{\text {Benzaldehyde }}+\mathrm{n}_{\text {Ethanol }}\right]$. LI as calculated as follows: $\mathrm{LI}=\mathrm{CI}_{\text {naive }}-\mathrm{CI}_{\text {trained }}$.

SL extraction. Extrachromosomal arrays of Psptl-1::SPTL-1::GFP and Psptl-1::SPTL-1(C121W)::GFP were integrated by gamma irradiation, and the integrated lines were outcrossed at least 4 times, and verified by single worm PCR followed by sequencing before harvesting for SL extraction. For collecting WT and transgenic worms, L4 stage worms were obtained by a standard bleaching-washing procedure to obtain a large population of embryos that were allowed to grow for $\sim 40-50 \mathrm{~h}$. The SPTL-1(C121W) transgenic animals grew slightly slower than SPTL-1 and N2 animals, and the worms were collected when $80 \%-90 \%$ were ascertained to be at L4 stage by visual inspection under the microscope. The $150 \mathrm{~mm}$ NGM culture plates were used throughout to grow a sufficiently large amount of synchronized worms. For each condition, $>800$ plates were harvested by rinsing the plates with M9 buffer and letting animals sediment at $4^{\circ} \mathrm{C}$. Worm pellets were subjected to three cycles of freezing in liquid nitrogen for 30-60 min and thawing on ice for $30 \mathrm{~min}$ followed by sonication ( $10 \mathrm{~s}$ each time, $3-5$ times for each cycle). The suspension was lyophilized before extraction to reduce the aqueous volume and for measurement of dry weight.

The worm samples (normally $30 \mathrm{mg}$ for each extraction) were suspended in $2 \mathrm{ml}$ of methanol and $1 \mathrm{ml}$ of chloroform in $13 \times 100-\mathrm{mm}$ screw-capped glass test tubes, prespiked with internal standards, lysed via sonication at room temperature, and incubated at $48^{\circ} \mathrm{C}$ for $24 \mathrm{~h}$, as described previously (Sullards et al., 2003). The supernatant was collected and dried under nitrogen at room temperature. The samples were digested by $75 \mu \mathrm{l}$ of $1 \mathrm{M} \mathrm{KOH}$ in methanol at $37^{\circ} \mathrm{C}$ for $2 \mathrm{~h}$, which is expected to reduce clogging of the LC columns, neutralized with $3 \mu \mathrm{l}$ of glacial acetic acid, and then back-extracted with $2 \mathrm{ml}$ of chloroform and $660 \mu \mathrm{l}$ of distilled, deionized water. After centrifugation at $2500 \mathrm{rpm}$ for $10 \mathrm{~min}$ to separate the phases, the upper layer was carefully removed and can be used for reextraction by adding 0.3 volume of water. The lower layer was dried under nitrogen and used for SL profile analysis. The SL standard mixture containing $25 \mu \mathrm{M}$ of different types of SL com- 
pounds was diluted 10-fold with chloroform:methanol 2:1 and then used for Liquid chromatography-tandem mass spectrometry (LCMS/MS) analysis.

LC-MS/MS-based SL profile analysis. The sphingolipidomics analysis was performed on a UPLC-MS/MS system equipped with a Xevo TQD tandem quadrupole mass spectrometer (Waters). The molecular subspecies of each category of SLs that is present were identified by their unique fragmentation products using precursor ion or neutral loss scans. The LC-MS method was built to monitor $>200$ compounds for which both the ionization and dissociation (collision energy) conditions have been optimized for individual molecular species. Given that $C$. elegans SLs are reported to mainly contain $\mathrm{d} 17: 1$ sphingoid bases, mass spectral optimization was performed under positive mode ionization to monitor precursor ion scanning for the $250.3 \mathrm{u}$ fragment of their common d17:1 sphingoid base, and sphingomyelin was optimized for its $[\mathrm{M}+\mathrm{H}]+$ $184.2 \mathrm{u}$ fragment. To identify the deoxy sphingoid bases and their ceramide derivatives, commercially available $\mathrm{m} 18: 0$ and $\mathrm{m} 17: 0$ were used as standard and the mass ions 286.3-268.3 (m18:0) and 272.3-254.3 (m17:0) for standard and 272.3-254.3 (m17:0) and 258.3-240.3 (m16:0) for worm samples were detected, respectively. To detect other sphingoid bases and their derivatives, the respective mass ions were optimized.

After optimizing the elution protocols for maximum recovery, a multiple reaction monitoring protocol was revised for quantitative measurement of $>80$ analytes across three periods in the triple-quad mass spectrometer. Almost all compounds, except Sa1P and So1P, were measured using normal-phase LC through a Waters ACQUITY UPLC BEH Amide column $(1.7 \mu \mathrm{m} 2.1 \times 100 \mathrm{~mm})$ with solution A $([97: 2: 1 \mathrm{ACN}$ : $\left.\left.\mathrm{CH}_{3} \mathrm{OH}: \mathrm{CH}_{3} \mathrm{COOH}\right]+5 \mathrm{~mm} \mathrm{NH}_{4} \mathrm{OAC}\right)$ and solution $\mathrm{B}\left(\left[99: 1 \mathrm{CH}_{3} \mathrm{OH}\right.\right.$ : $\left.\mathrm{CH}_{3} \mathrm{COOH}\right]+5 \mathrm{~mm} \mathrm{NH}_{4} \mathrm{OAC}$ ) as mobile phases. The flow rate was set at $0.4 \mathrm{ml} / \mathrm{min}$, with the gradient setting as $0-1 \mathrm{~min}, 100 \% \mathrm{~A} ; 1-4 \mathrm{~min}$, $100 \%-0 \% \mathrm{~A} ; 4-8 \mathrm{~min}, 100 \% \mathrm{~B} ; 8-10 \mathrm{~min}, 100 \%-0 \% \mathrm{~B} ; 10-12 \mathrm{~min}$, $100 \%$ A. SalP and SolP were analyzed using reverse-phase LC through a Waters ACQUITY UPLC BEH C18 column $(1.7 \mu \mathrm{m} 2.1 \times 100 \mathrm{~mm})$ with solution A consisted of $0.1 \%$ formic acid in ACN/water $(20: 80, \mathrm{v} / \mathrm{v})$ and B consisted of $0.1 \%$ formic acid in ACN/2-propanol (20:80, v/v) as mobile phases. The flow rate was set at $0.25 \mathrm{ml} / \mathrm{min}$, with the gradient setting as $0-1 \mathrm{~min}, 70 \% \mathrm{~A} ; 1-2.5 \mathrm{~min}, 60 \%-30 \% \mathrm{~A} ; 2.5-4 \mathrm{~min}, 30 \%-20 \% \mathrm{~A}$; 4-5 min, 20\% A; 5-6.5 min, 20\%-10\% A; 6.5-6.6 min, 10\%-0\% A; $6.6-8 \mathrm{~min}, 100 \% \mathrm{~B}$. Concentrations for each compound were calculated from the response ratio of compound area/internal standard area of the corresponding period per dry weight of the sample.

Dissecting fluorescence and confocal microscopy. To evaluate the morphological defects in intestine and neurons, live animals were directly observed on plates under an SMZ180 microscope (Nikon) equipped with a high-power stereo fluorescent attachment and a digital CCD camera. To observe subcellular localization of the intestinal and neuronal proteins in detail, live worms were mounted on glass slides and anesthetized using $10 \mathrm{~mm}$ sodium azide (Sigma-Aldrich) and visualized by an LSM 710 confocal microscope (Carl Zeiss). Single-plane images were taken as $6-50$ sections along the $z$ axis at $0.2 \mu \mathrm{m}$ intervals. $Z$ stacks were collected and then merged into a single projection image with the maximum intensity for additional analysis. Multichannel images were taken after adjusting individual channels to eliminate bleed-through and sequentially scanned. Images were taken at minimal laser settings where not indicated otherwise. Identical laser and confocal settings were used when comparing experimental animals with controls. Images were arranged using Adobe Photoshop with occasional small adjustments for contrast and brightness.

Statistical analysis. Sphingolipidomics data were analyzed using Metaboanalyst 4.0 (RRID:SCR_015539) (Chong et al., 2018), and other results were analyzed using Prism software (GraphPad Software, RRID: SCR_002798) and are presented as mean \pm SD unless otherwise stated. Statistical analyses were performed with the aid of the two-tailed Student's $t$ test for between-group comparisons, one-way ANOVA with Tukey's multiple-comparisons test for comparisons among three or more groups, and two-way ANOVA analysis with Tukey's multiplecomparisons test for comparisons between groups on two independent variables. A $p$ value $<0.05$ was considered to reflect statistical signifi- cance. All data are representative of those of at least three independent experiments unless otherwise indicated.

\section{Results}

sptl-1(c363g) homozygotes, but not heterozygotes, display the same larval lethality and epithelial polarity defects as sptl-1(RNAi) animals

Protein sequence alignment shows that the human and C. elegans homologs of SPTLC1 share 50\% identity, and C133 (C121 in C. elegans) is located within a highly conserved protein domain. We used CRISPR-Cas9-mediated genome editing to generate a sptl$1(c 363 g)$ mutation, encoding SPTL-1(C121W) and equivalent to human SPTLC1 ${ }^{\mathrm{C} 133 \mathrm{~W}}$, at its genomic locus. We constructed transgenic animals that ubiquitously expressed Cas9 under the control of an eft-3 promoter and one single-guide RNA driven by the U6 gene promoter. While screening for animals carrying the sptl-1(c363g) mutation by single-worm PCR, we found that all F2 progeny checked $(n>100)$ displayed either a WT or a heterozygous genotype. Endonuclease-based assays and sequencing results demonstrated that these mutants generated the expected molecular lesion but carried only one mutant allele (Fig. 1A,B). Next, we observed $\sim 10 \%-20 \%$ dead larval progeny from heterozygous parents, suggesting that homozygous sptl-1(c363g) mutants are larval lethal, consistent with the sptl-1(RNAi) phenotype (Zhang et al., 2011). After introducing ERM-1::GFP, an apical membrane marker in intestine, into the mutant strain, we found that sptl-1(c363g) homozygous mutants phenocopied sptl1(RNAi) animals, exhibiting the signature apicobasal polarity conversion and ectopic lumen phenotype in the C. elegans intestine at the L1 stage (Fig. 1C), whereas the heterozygous animals looked WT. Together, these results strongly suggested that SPTL$1(\mathrm{C} 121 \mathrm{~W})$ exerted a loss-of-function effect.

To examine whether loss of SPTL-1 function is responsible for the growth phenotypes, we expressed full-length sptl-1 cDNA fused with a GFP coding sequence under the control of the sptl-1 promoter (part of the sequence containing c363 shown in Fig. $2 A)$. GFP fluorescence was detected in the intestine as well as some unidentified cells of the head from early embryonic stage throughout development. In intestinal cells, SPTL-1::GFP displayed a cytoplasmic punctate, yet clear perinuclear, pattern during embryogenesis and developed into a predominantly cytoplasmic punctate pattern in larvae and adults (Fig. $2 B, C$ ). When introduced into sptl-1(c363g) mutants, SPTL-1::GFP was able to fully rescue the mutant, establishing a homozygous sptl$1(c 363 g)$ strain balanced by the transgene (Fig. 2D). This result is consistent with a loss-of-function effect of the mutation and argues against a gain-of-function scenario or a toxic product as the cause of the phenotype. Moreover, SPTL-1::GFP restored epithelial polarity and larval survival regardless of the diversity of expression patterns due to the heterogeneity of extrachromosomal arrays (Fig. 2D,F). For example, both the worms with GFP detected in whole or parts of intestines (Fig. 2D, top right) and the worms with GFP only in several head neurons (Fig. $2 D$, bottom right) were able to survive and grow into adults, suggesting that SPTL-1 regulates growth and epithelial polarity cell nonautonomously. To examine this possibility, we expressed the sptl-1 cDNA exclusively in the nervous system using a pan-neuronal rgef-1 promoter. This transgene also fully rescued the epithelial polarity defects and larval lethality and established a transgene balanced homozygous strain (Fig. 2E,F). Collectively, these results suggest that SPTL-1 acts cell nonautonomously in regulating epithelial polarity and growth, probably because of the ability 
A

B

$$
\begin{array}{ll}
s p t l-1 & \rightarrow \text { GAAACAGACGATTTTCAAGTACGGCGTAGGATCGTGCGGGCCACGTGGA } \\
\text { sptl-1 (c363g) } & \rightarrow \text { GAAACAGACGATTTTCAAGTACGGCGTAGGATCC GGGGCOCCGTGGA }
\end{array}
$$

\section{Heterozygote:}
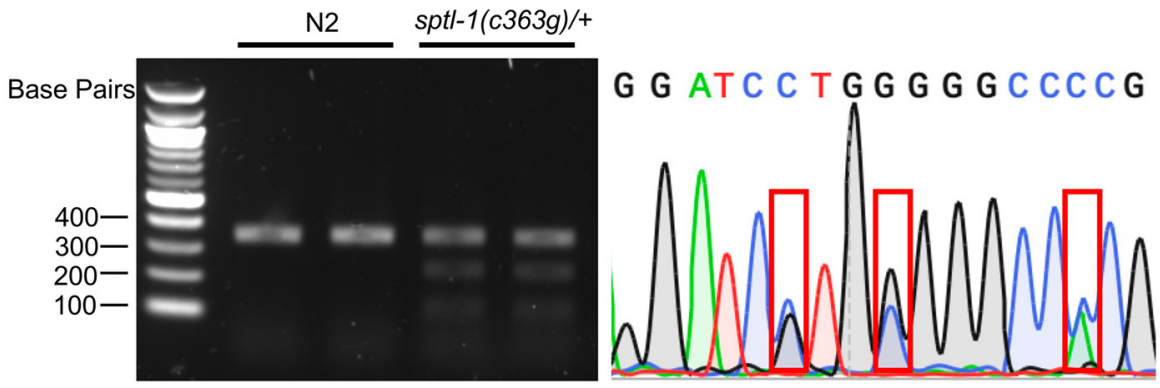

Homozygote:
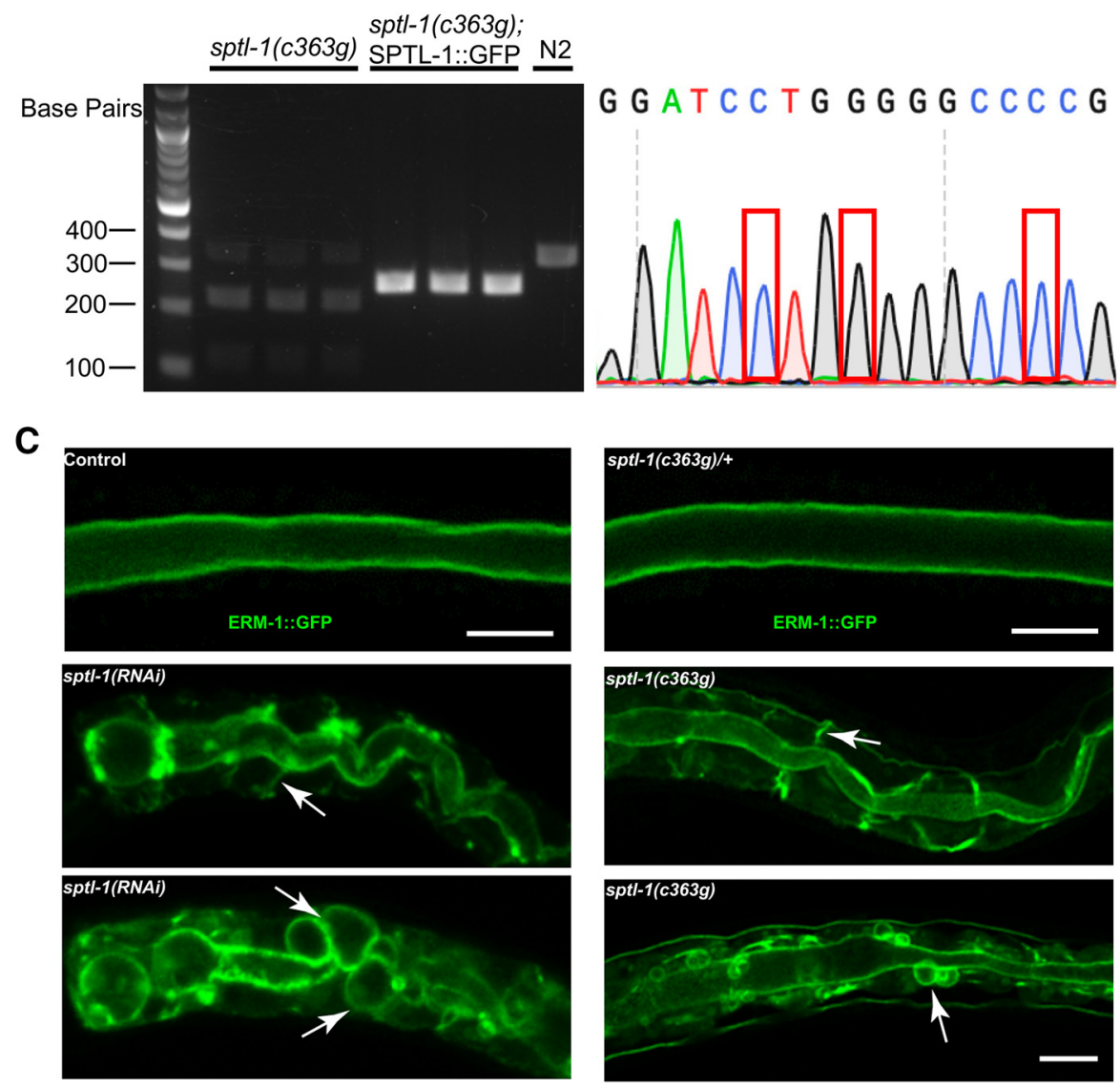

Figure 1. sptl-1(c363g) homozygous animals exhibit larval lethality and epithelial polarity defects. A, sptl-1(c363g) mutants were generated by CRISPR. Alignment of the DNA sequence of the C. elegans WT sptl-1 gene and the donor oligonucleotide designed for CRISPR: carrying the sptl-1(c363g) mutation (red), and two additional silent mutations (blue) to ablate the gRNA cleavage site and to introduce restriction sites for ease of screening. $\boldsymbol{B}$, Genotype verification for spt-1/(c363g)/+ and sptl-1(c363g) by PCR followed by restriction digestion. Heterozygote: Adult-stage single-worm PCR. Single band at $\sim 350$ bp indicated WT, and triple bands indicated heterozygosity. Homozygote: L1-stage multiworm PCR. Triple bands indicated homozygosity (faint WT band is due to incomplete restriction digestion), whereas homozygous animals carrying transgene showed single band derived from transgene as template. Transgenic animals and N2 generated the DNA fragments with different sizes due to difference in the templates (CDNA vs genomic DNA). The spectrum of sequencing results showing the heterozygosity and homozygosity at the designated sites. C, Confocal section images of control, sptl-1(RNAi), and sptl-1(c363g) heterozygous and homozygous mutant animals expressing ERM-1::GFP. WT apical localization (top), ectopic basolateral staining (middle, arrow) of ERM-1::GFP and ectopic lumens (bottom, arrow). Scale bars, $5 \mu \mathrm{m}$.

of sphinganine, the immediate product of SPTL-1, to move freely between different cells (Merrill and Sandhoff, 2002).

We then examined whether dietary supplementation of sphinganine could rescue the lethality phenotype. C. elegans contains a unique d17iso-sphinanine, synthesized through condensation of the monomethyl branched chain fatty acid C15ISO with serine (Chitwood et al., 1995). The custom-synthesized di7isosphinganine was able to partially rescue the larval lethality and 
A

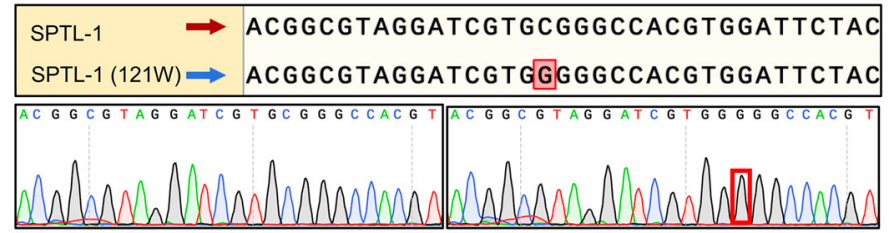

B
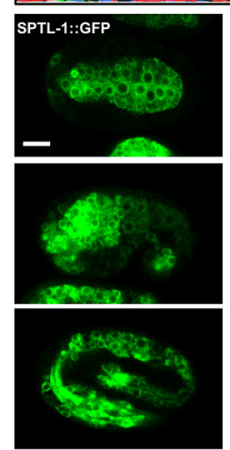

D
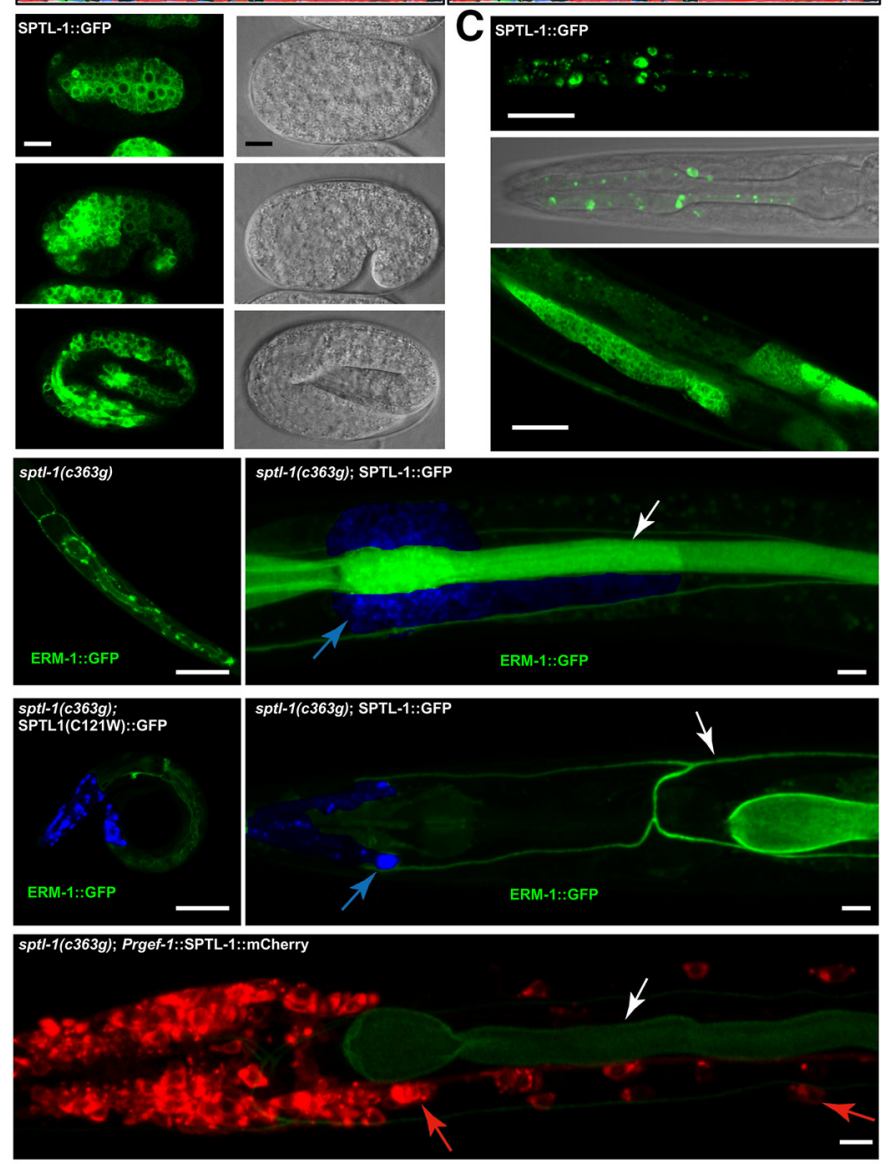

$\mathbf{F}$

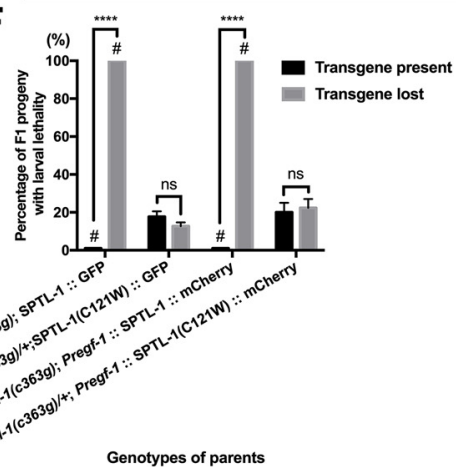

G

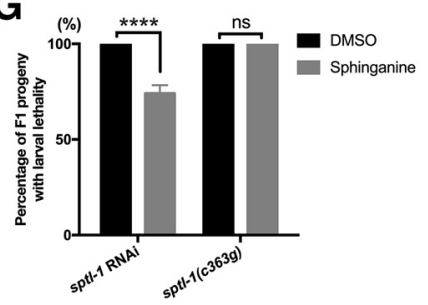

Genotypes of progeny

Genotypes of parents

Figure 2. sptl-1(c363g) homozygote phenotypes can be rescued by overexpression of SPTL-1, but not SPTL-1(C121W), transgenes. $\boldsymbol{A}$, Genotype verification of SPTL-1::GFP and SPTL-1(C121W)::GFP integrated strains by single-worm PCR followed by sequencing. Red represents (363g mutation. B, Localization of SPTL-1::GFP in bean (top), comma (middle), and threefold stage of embryos (bottom) shown. $\boldsymbol{C}$, Expression of SPTL-1::GFP in some head neurons (top, fluorescence image; middle, overlay with differential interference contrast) and cytoplasm of intestine (bottom) in larval worms. D, Larval arrest and epithelial polarity defects of sptl-1(c363g) mutants (left top) are rescued by expressing SPTL-1 (right), but not by SPTL-1(C121W) (left bottom), driven by an sptl-1 (Psptl-1) promoter. White arrow indicates ERM-1::GFP. Blue arrow indicates SPTL-1::GFP. Rescued animals with SPTL-1::GFP only detected in a few intestinal cells (right top) or in a few neurons (right bottom) shown. SPTL-1::GFP is pseudocolored blue for clarity. E, Pan-neuronal expression (Prgef-1) of SPTL-1 rescues sptl-1(c363g) mutant phenotypes. Red arrow indicates SPTL-1::mCherry. White arrow indicates ERM-1::GFP. F, Transgenic SPTL-1, but not SPTL-1(C121W), fully rescues the L1 intestinal phenotype. F1 progeny shown on $y$ axis; parent genotype on $x$ axis. In bars marked with \#, F1s are derived from homozygous parents because the SPTL-1 transgene allows the propagation of a sptl-1(c363g) homozygous strain. No such strain can be generated by SPTL-1(C121W) (all homozygous progeny, with and without the transgene, dies as larvae); therefore, F1s are derived from sptl-1(c363g)/+ heterozygous parents in all other bars. The intestinal phenotype and larval lethality are closely correlated: almost all the animals with intestinal polarity defect at the $\mathrm{L} 1$ stage die as larvae. $F_{(3,16)}=672.9$. $^{* * *} p<0.0001$ (two-way epithelial polarity defects of sptl-1 RNAi animals, confirming previous results (Fig. $2 G$ ) (Zhu et al., 2013). However, no rescue was observed for the homozygous progeny of $s p t l-1(c 363 g) /+$ or $s p t l-1(c 363 g)$; SPTL-1::GFP animals, even when fed with sphinganine through several generations. We attribute this failure to the inability of external sphinganine to compensate for the reduced amount of maternal lipids that is transmitted to the progeny of germline mutant compared with RNAi-treated parents and that may be prohibitive for the survival of progeny lacking any WT sptl-1 allele. Indeed, despite the documented requirement for maternal spingolipids (Kuervers et al., 2003; Nomura et al., 2011; Zhang et al., 2011), the sptl-1 L1 RNAi phenotype was found to be less severe than that of the homozygous progeny of $s p t l-1(c 363 g) /+$ or sptl-1(c363g);SPTL-1::GFP animals (data not shown), suggesting that a higher amount of maternal lipids makes up for the loss of maternal RNA in these animals that would otherwise be expected to have a more severe phenotype.

\section{sptl-1(c363g)/+ heterozygous animals} show growth and behavioral defects Although sptl-1(c363g) homozygous mutants are arrested at the L1 stage, the heterozygous animals appear grossly normal, except with a small but significant decrease in brood size (Fig. 3A), consistent with a known role of SLs in fertility (Nomura et al., 2011). We next examined the effect of the mutation on life span. As shown in Figure $3 B-D$, the maximum, the median, and the mean life spans of the sptl-1(c363g)/+ heterozygous mutants were shortened compared with control animals (Fig. $3 B-D$ ), indicating that SLs have an impact on longevity. Given that HSAN1 patients carrying one SPTLC1 ${ }^{\mathrm{C} 133 \mathrm{~W}}$ allele show severe distal neuronal sensory loss and motor involvement, neuronal and behavioral defects were evaluated in sptl-1(c363g)/+ heterozygous animals. We first checked

ANOVA followed by Tukey HSD post hoc test). ns (not significant, $p>0.05$ ) Error bars indicate mean \pm SEM. G, Quantification of phenotype restoration by d17iso:0 sphinganine of sptl-1/(c363g) and sptl-1 RNAi animals. For sptt-1(c363g) genotype, homozygous progeny from sptt-1((363g)/+ heterozygous parents or nontransgenic homozygous progeny from sptt-1((363g);SPTL-1::GFP parents were scored and ns (not significant, $p>0.05$ ). $F_{(1,8)}=106.7$. ${ }^{* * * *} p<0.0001$ (twoway ANOVA followed by Tukey HSD post hoc test). Error bars indicate mean \pm SEM. Scale bars, $5 \mu \mathrm{m}$. $F$, G, Three independent experiments were performed with at least 100 worms examined in each. 

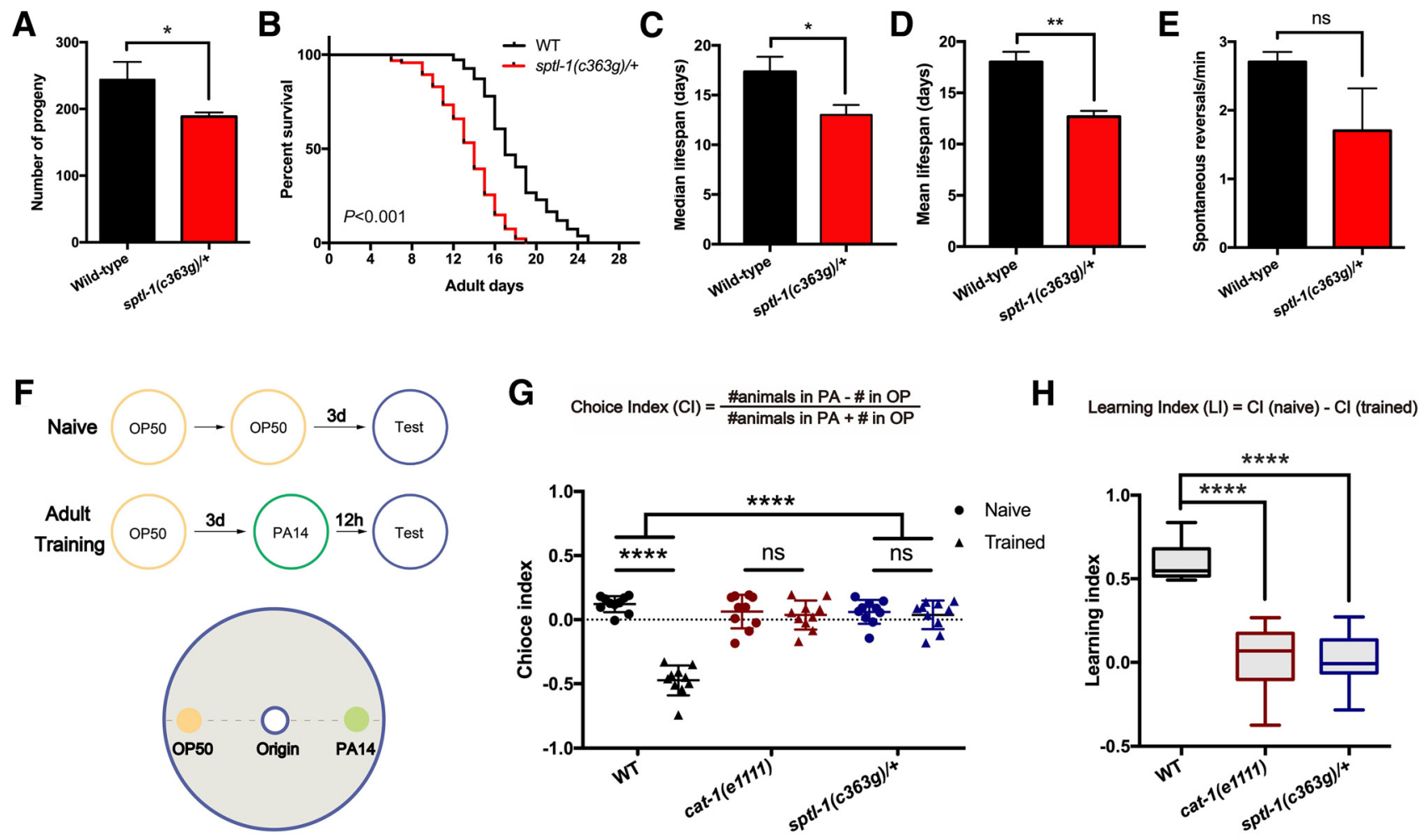

Learning Index $(\mathrm{LI})=\mathrm{Cl}$ (naive) $-\mathrm{Cl}$ (trained)
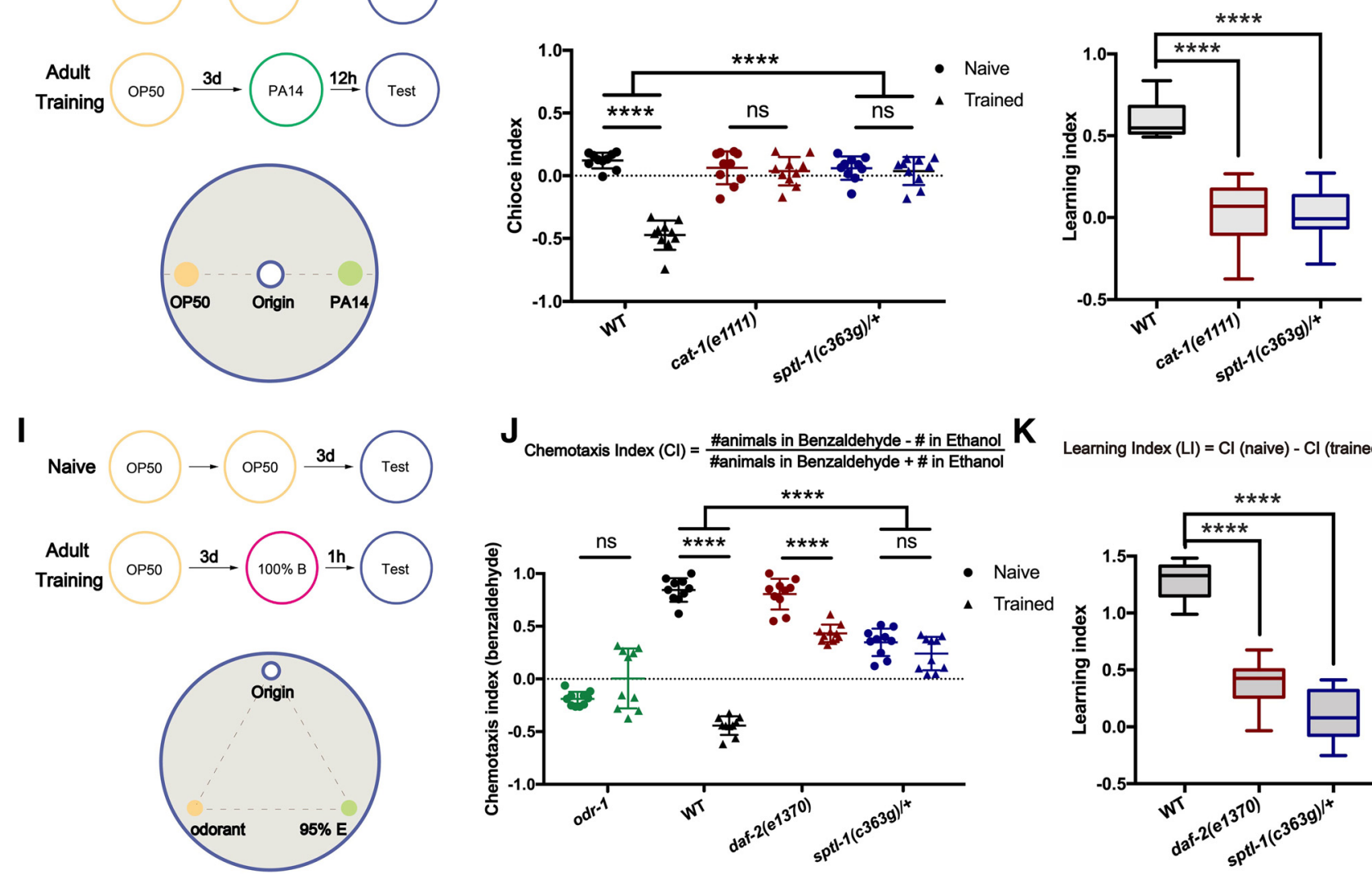

Figure 3. spt-1(c363g)/+ heterozygous animals show growth and behavioral defects. Compared with WT, sptl-1((c363g)/+ heterozygous animals display reduced brood size $(\boldsymbol{A})$, shortened life span $(\boldsymbol{B}-\boldsymbol{D})$, marginally decreased reversal frequency $(\boldsymbol{E})$, impaired aversive olfactory learning toward pathogenic bacteria PA14, cat-1(e1111), as positive control $(\boldsymbol{F}-\boldsymbol{H})$, and chemical odor benzaldehyde, odr-1(n1936), as positive control for odorant chemotaxis, daf-2(e1370), as positive control for associative learning (I-K).F,I, Training protocol and schematics for aversive olfactory learning assays. Three independent experiments were performed with at least 100 worms scored in each for $\boldsymbol{A}-\boldsymbol{E}$ and $>1000$ for $\boldsymbol{F}-\boldsymbol{K}$. $\boldsymbol{A}-\boldsymbol{E}$, Genotypes of each scored animal were determined either through the presence of epithelial polarity defect in the F1 progeny or by single-worm PCR followed by restriction digestion. $G, J$, Each dot represents a single population assay of $100-200$ animals. Each line indicates the mean value. $\boldsymbol{A}, \mathbf{C}-\boldsymbol{E}$, WT and sptl-1(c363g)/+ groups were compared using two-tailed Student's $t$ test. Data are mean \pm SD. ${ }^{*} p<0.05 .{ }^{* *} p<0.01$ and ns (not significant, $p>0.05$ ). B, Statistical significance was determined by log-rank (Mantel-Cox) test for the survival curves as shown $(p<0.001)$. G, J, Statistical analysis was conducted using two-way ANOVA, Tukey HSD post hoc test: $F_{(2,54)}=29.57(G) ; F_{(2,54)}=127.1(J)$. Error bars indicate mean \pm SEM. ${ }^{* * * *} p<0.0001$ and ns (not significant, $\left.p>0.05\right)$. $\boldsymbol{H}, \boldsymbol{K}$, Statistical analysis was conducted using one-way ANOVA, Tukey HSD post hoc test: $F_{(2,27)}=45.22(\boldsymbol{H}) ; F_{(2,27)}=98.79(\boldsymbol{K})$. Error bars indicate mean \pm SEM. ${ }^{* * * *} p<0.0001$.

locomotion defects by counting body bends, $\omega$ turns, and spontaneous reversal frequency, and detected some, however, not statistically significant, differences between WT and sptl-1(c363g)/+ heterozygous animals (Fig. 3E). Interestingly, $>80 \%$ of these sptl1(RNAi) L4 larvae, treated with mild RNAi to survive beyond the L1 stage (see Materials and Methods), exhibited mild intestinal polarity defects as well as uncoordinated locomotion after $3 \mathrm{~d}$ treatment with RNAi (data not shown), suggesting that SLs are important for locomotion behavior. We next examined the function of SLs in learning and memory. This assay was based on $C$. elegans' ability not only to distinguish between nutritious and pathogenic bacteria, such as Pseudomonas aeruginosa PA14, but also to memorize the experience and learn to avoid the bacterial odor (Jin et al., 2016). WT animals displayed a clear shift in bacterial preference in response to $12 \mathrm{~h}$ adult-stage conditioning. sptl-1(c363g)/+ heterozygous mutants showed abolished bacterial preference and reduced pathogen aversion, similar to that of cat-1(e1111) (RRID:WB-STRAIN:CB1111) mutants (Zhang et al., 2005) (Fig. 3F-H). Given that the PA14 conditioning program neither affected the dwelling behavior nor altered intestinal morphology of $s p t l-1(c 363 g) /+$ heterozygotes (data not shown), the reduced pathogen aversion strongly suggests a defect in sen- 
sory neuron function. To further confirm the role of SLs in sensory function, we examined the worms' associative learning in the presence of other chemical odors, such as benzaldehyde. Similarly, the sptl-1(c363g)/+ heterozygous animals exhibited impaired olfactory response to benzaldehyde, albeit to a lesser extent compared with odr-1(n1936) (RRID:WB-STRAIN: CX2065) mutants (Bargmann et al., 1993), and are also defective in benzaldehyde-starvation associative learning, similar to daf2(e1370) (RRID:WB-STRAIN:CB1370) animals (Lin et al., 2010) (Fig. 3I-K). Our results demonstrate that SLs are required for responses to specific odorants and thus for sensory functions of neurons and reveal that sptl-1(c363g)/+ animals display characteristics of neuronal dysfunction seen in patients with HSAN1.

\section{sptl-1(c363g)/+ heterozygous animals exhibit defects in neuronal morphology and polarity}

We next examined the morphology of the nervous system of sptl-1(c363g)/+ animals and assessed neuron fate and polarized outgrowth of axons and dendrites in animals marked with Punc25::gfp (RRID:WB-STRAIN:CZ1200) (Huang et al., 2002), which labels all GABAergic neurons. Normally, the 6 DD and 13 VD neurons in the ventral body region send circumferential commissures that reach the dorsal cord during embryogenesis or larval development (White et al., 1986). WT L4-stage animals maintain a dorsal-ventral path for each individual commissure (Fig. 4A). To investigate the effect of the sptl-1(c363g)/+ genotype on neuronal morphology, we assessed the progeny of $s p t l-1(c 363 g) /+$ parents. In sptl-1 (c363g)/+ L4-stage larval progeny (a population containing two-thirds heterozygotes and one-third WT animals), there was no alteration in neuron number and fate; however, many commissures of DD and VD neurons either changed orientation or ectopically branched or showed occasional premature arrest (Fig. $4 B, C$ ) as previously reported (Teulière et al., 2011), suggesting a defect in polarized outgrowth of axons and dendrites. This result indicates that dysfunction of SPTL-1, and thereby aberrant SL metabolism, affects polarized axon outgrowth and guidance.

Neurons have distinct subcellular domains with axons specified by Par3/Par6/aPKC complex components functioning to send out electrochemical signals, and dendrites characterized by a different set of protein components acting to receive the signals (Bentley and Banker, 2016). SLs have been shown to play essential roles in determining apicobasal polarity in epithelial cells (Zhang et al., 2011). To investigate the role of SLs in neuronal polarity in C. elegans, we visualized synaptic vesicle localization in live animals using a GFP-tagged synaptobrevin (SNB-1::GFP), a transmembrane synaptic vesicle protein. We first examined the localization of SNB-1::GFP in the GABAergic DD motor neurons. In $\mathrm{L} 1$ larvae, the dorsal processes of the $\mathrm{DD}$ neurons receive signals from DA and DB excitatory motor neurons, acting as postsynaptic terminals (dendrites), and the ventral processes form en passant synapses to ventral body wall muscles, serving as presynaptic terminals (axons), whereas from the L2 stage onward, the synaptic polarity of DD neurons is reversed (White et al., 1978). In WT L1 larvae, Punc-25::SNB-1::GFP is restricted to the presynaptic ventral DD processes (Fig. 4D). By contrast, $\sim 15 \%$ of L1-larval progeny of $s p t l-1(c 363 g) /+$ parents (a population containing 50\% sptl-1(c363g)/+ animals) displayed numerous ectopic GFP signals along the dorsal nerve cord (Fig. $4 E, F)$, suggesting a neuronal polarity defect.

We also examined the polarized distribution of SNB-1 vesicles in other neurons. RIA neurons have one bipolar neurite, consisting of a distal region acting as axon, a proximal region acting as dendrite, and an isthmus-like structure devoid of synapses in between (White et al., 1986) (Fig. 4G). In L4 stage WT animals, SNB-1::GFP driven by $g l r-3$ promoter (RRID:WB-STRAIN: IK721) (Tanizawa et al., 2006) was localized exclusively to the distal presynaptic (axonal) region in a punctate pattern, indicating that SNB-1 correctly localized to presynaptic vesicles. In contrast, SNB-1 was mislocalized throughout the entire length of the neurite (designated as Type I defect; Fig. 4H,I) and/or accumulated in large puncta in presynaptic regions in L4 progeny of sptl-1(c363g)/ + animals (designated as Type II defect; Fig. 4J, K). The ASI neurons have cell bodies near the nerve ring, an axonal process wrapping around the nerve ring, and a dendritic process extending all the way to the nose tip (White et al., 1986). In each ASI neuron of L4-stage animals carrying str-3-driven SNB-1:: GFP (RRID:WB-STRAIN:CX3572) (Crump et al., 2001), GFP fluorescence is visible in several regularly spaced clusters along the distal half of the axon in the nerve ring (Fig. $4 L$ ). GFP is also weakly present in the cell body, and occasionally in the dendrite of some animals in a diffuse pattern. In contrast, L4 progeny of sptl-1(c363g)/+ parents displayed highly penetrant SNB-1::GFP defects in ASI. In some animals, some regions of the axon have densely packed vesicle clusters interspaced with diffusing or reduced SNB-1::GFP fluorescence (defined as Type I defect; Fig. $4 M, N$ ), whereas in other animals, weak SNB-1-positive vesicle clusters are observed in dendrites (defined as Type II defect; Fig. $4 O, P)$. Most animals exhibit a combination of these defects.

Importantly, sptl-1(RNAi) L4 larvae (treated with mild RNAi) displayed, in addition to uncoordinated locomotion, neuronal morphology and polarity defects in DD, RIA, and ASI neurons similar to sptl-1(c363g)/+ heterozygous mutants (Fig. 4). Thus, neuronal defects also appear to be caused by a loss-of-function effect of the SPTL-1(C121W) mutation.

Together, these results demonstrate that SLs play important roles in maintaining axon-dendrite identity and affect polarized trafficking in the nervous system. In contrast to the recessive effect of $s p t l-1(c 363 g)$ on intestinal polarity, SPTLC1 ${ }^{\mathrm{C} 133 \mathrm{~W}}$ derived dominant effects on the nervous system of heterozygous animals may reflect particular susceptibility of neurons to a subtle reduction in SL biosynthesis.

\section{SPTL-1(C121W) acts in a dominant-negative manner in the C. elegans intestine and nervous system to alter cellular functions}

To further examine the dominant effect of the HSAN1 mutation, we characterized our transgenic lines overexpressing the SPTL$1(\mathrm{C} 121 \mathrm{~W}):$ :GFP fusion protein [SPTL-1 (C121W) OE] under the control of either the sptl-1 promoter or a pan-neuronal rgef-1 promoter. No difference was detected in localization or fluorescence intensity between SPTL-1 and SPTL-1(C121W) in the intestine or nervous system (Fig. $5 A, F$ ). Mutant proteins also did not seem to form toxic protein aggregates. In contrast to full rescue of the $s p t l-1(c 363 g)$ intestinal phenotype and lethality by WT SPTL-1, expression of the mutant protein resulted in no rescue (Fig. 2D,F). Moreover, SPTL-1(C121W) OE, but not overexpression of SPTL-1::GFP (SPTL-1 OE), driven by their own promoter (mainly in the intestine), induced 10\%-15\% larval lethality with an intestinal phenotype similar to the late-stage severe sptl-1(c363g) and sptl-1(RNAi) epithelial polarity defect (Fig. $5 B, C$ ). Overexpression of pan-neuronal SPTL-1(C121W), but not SPTL-1, also recapitulated the sptl-1(c363g)/+ and sptl1(RNAi) axonal outgrowth and RIA polarity defects (Fig. 5G-J). Moreover, $\mathrm{P}_{\text {sptl-1 }}$ SPTL-1(C121W) OE induced a mild intestinal polarity phenotype in $>50 \%$ of otherwise WT-appearing sptl- 

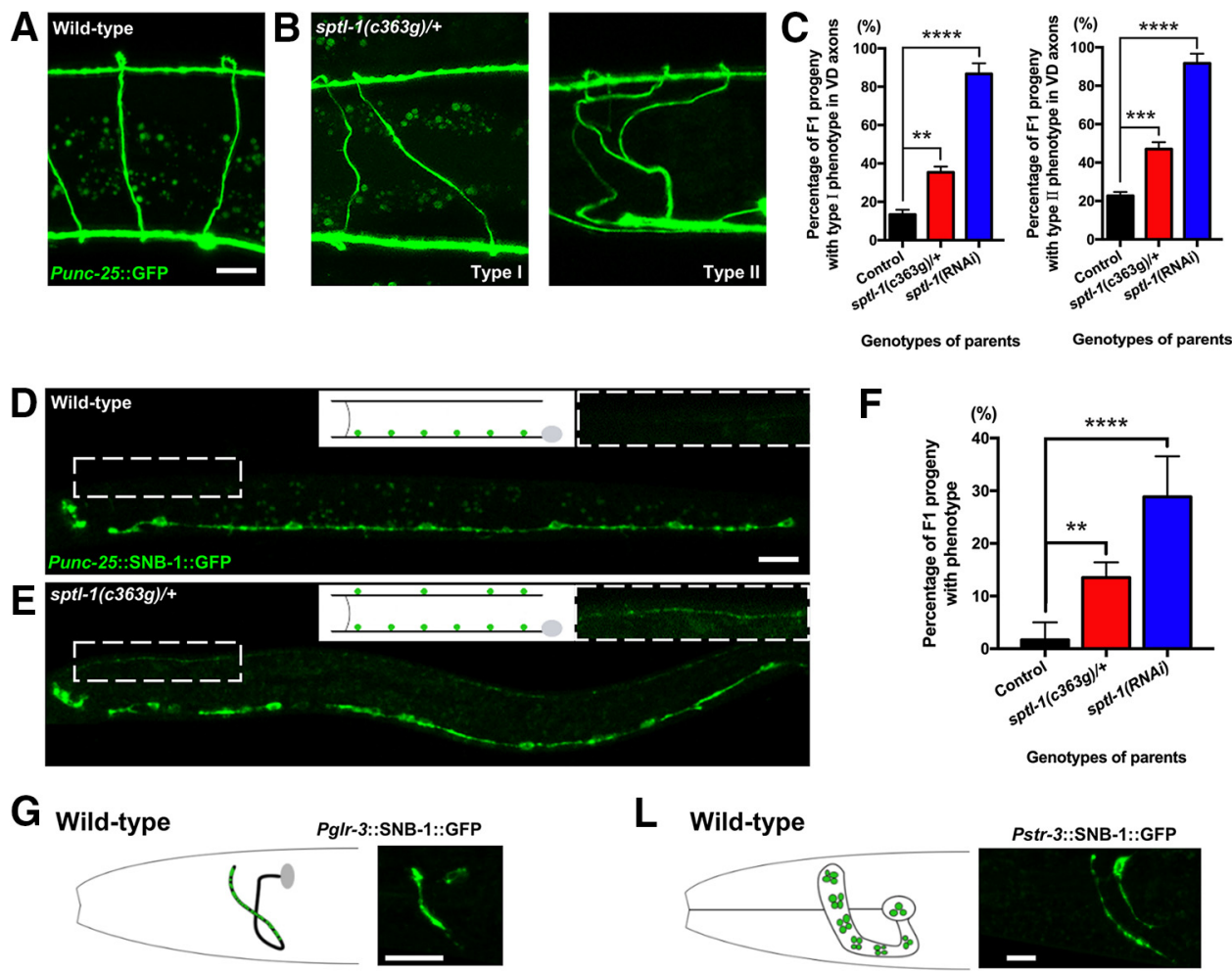

Genotypes of parents

Genotypes of parents

F

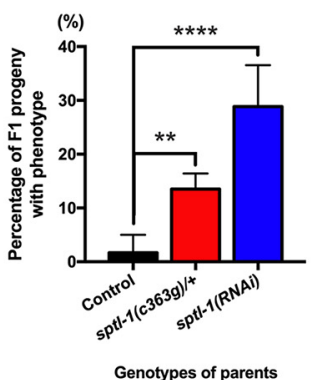

L Wild-type

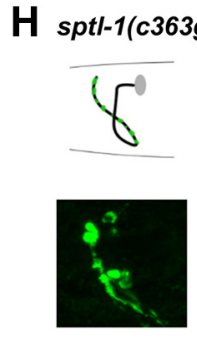

Type I

J $s p t l-1(c 363 g) /+\quad K$

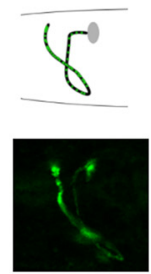

Type II

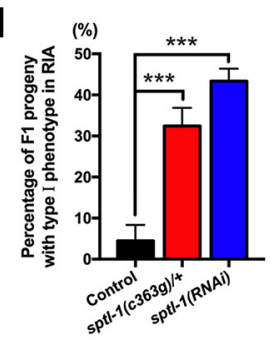

Genotypes of parents

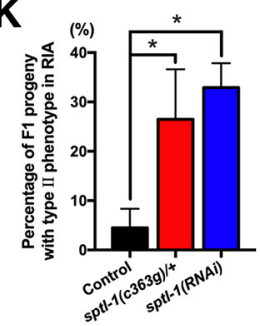

Genotypes of parents
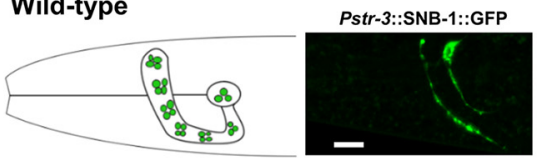

M sptl-1(c363g)/+
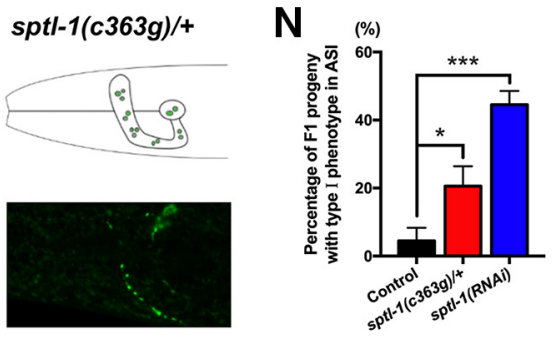

Type I

O sptl-1(c363g)/+

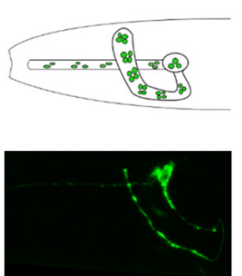

Type II
Genotypes of parents

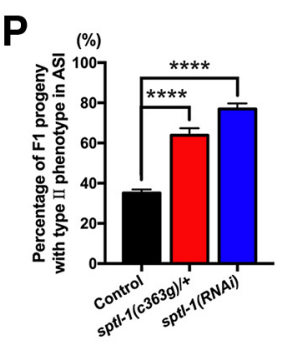

Figure 4. sptl-1(c363g)/+ heterozygote animals have neuronal morphology and polarity defects. $\boldsymbol{A}-\boldsymbol{C}$, Defects in VD/DD axon morphology. Normal $(\boldsymbol{A})$ and defective (B) VD/DD commissural trajectories. Type I: commissures deviating from the normal dorsal-ventral orientation by $>45^{\circ}$; Type Il: extra branches for one commissure (Teulière et al., 2011). The severity of phenotypes varies with animals. $\boldsymbol{D}-\boldsymbol{F}$, Defects in axon-dendrite identity of L1 DD neurons. SNB-1::GFP localization in ventral processes of DD neurons in WT L1s (D), and in both ventral and dorsal processes in sptl-1(c363g)/ + L1s $(\boldsymbol{E})$ shown. Insets, Boxed areas are magnified 2X. G-K, Defects in axon-dendrite identity of RIA neurons. Exclusive SNB-1 localization in the presynaptic vesicles in WT (G), and accumulation of SNB-1 into big puncta in the presynaptic region (Type I phenotype in RIA neurons) $(\boldsymbol{H})$, and mislocalization in the postsynaptic region (Type II phenotype in RIA neurons) ( () in mutants shown. $\boldsymbol{L}-\boldsymbol{P}$, Defects in axon-dendrite identity of ASI neurons. SNB-1 localization in the axons of ASI neurons in WT animals $(\boldsymbol{L})$, fewer puncta in axons (Type I phenotype in ASI neurons) ( $\boldsymbol{M}$ ), and mislocalization in the dendrite (Type Il phenotype in ASI neurons) (O) in mutants shown. $\boldsymbol{C}, \boldsymbol{F}, \boldsymbol{I}, \boldsymbol{K}, \mathbf{N}, \boldsymbol{P}$, Quantifications for the phenotypes in different backgrounds. Of note, in theory, one-fourth of WT and one-fourth of homozygous animals for the heterozygous group in $\boldsymbol{F}$, and one-third of WT animals for the heterozygous groups in $C, F, I, K, N$, and $\boldsymbol{P}$ were included in the quantification. Therefore, the real penetrance may be higher. Three independent experiments were performed with at least 200 worms scored in each. Scale bars, $10 \mu \mathrm{m}$. Data are mean $\pm S D$. Statistical analysis was conducted using the one-way ANOVA, Tukey HSD post hoc test: $F_{(2,6)}=277.1, F_{(2,6)}=258.3, p<0.0001(\boldsymbol{C}) ; F_{(2,10)}=32.67, p<0.0001(\boldsymbol{F}) ; F_{(2,6)}=66.35, p<0.0001(\boldsymbol{I}) ; F_{(2,6)}=10.12$. $p=0.0119(K) ; F_{(2,5)}=42.01 . p=0.0007(\boldsymbol{N}) ; F_{(2,6)}=203.5, p<0.0001(\boldsymbol{P}) .{ }^{*} p<0.05,{ }^{* * *} p<0.01,{ }^{* * *} p<0.001,{ }^{* * * *} p<0.0001$. 

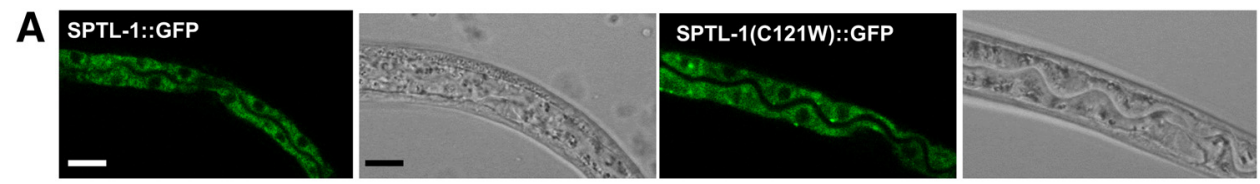

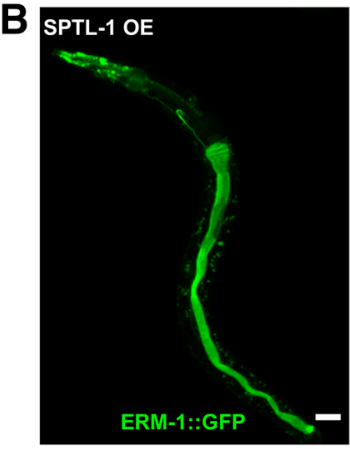

D

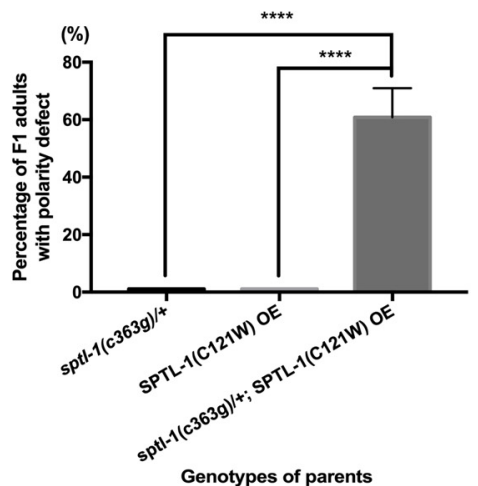

Genotypes of parents
C

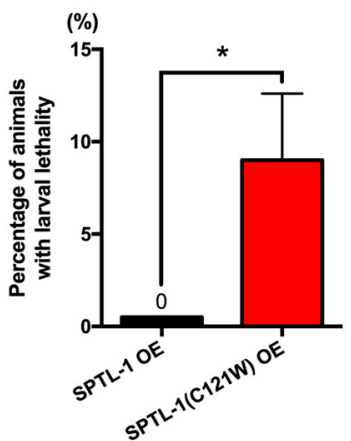

\section{E}
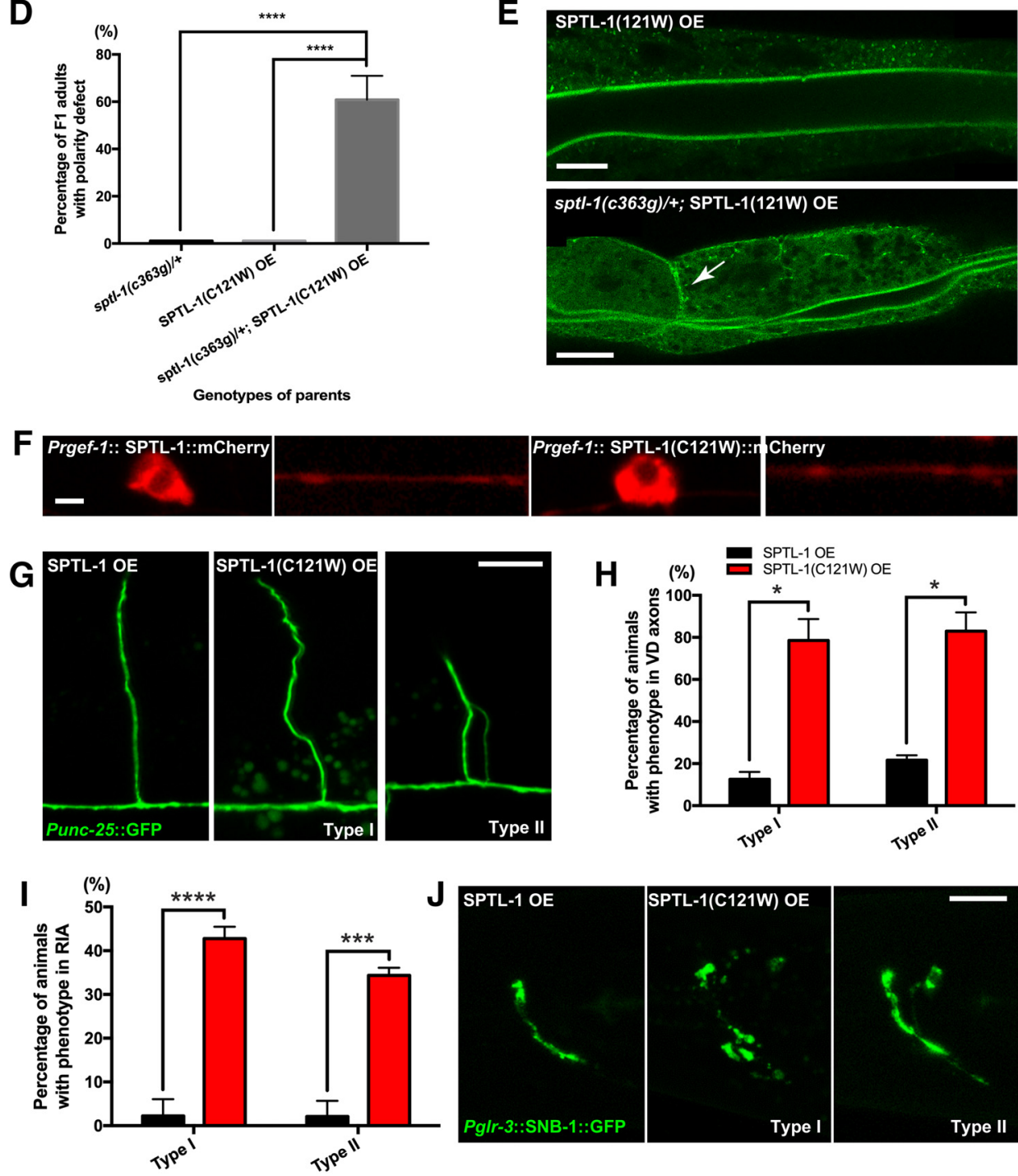

Figure 5. SPTL-1(C121W) acts in a dominant-negative manner in the C. elegans intestine and nervous system. $A$, Localization of SPTL-1::GFP and SPTL-1(C121W)::GFP in larval intestines. $B, C$, Overexpression of SPTL-1(C121W), but not SPTL-1, driven by an sptl-1 promoter, leads to intestinal morphology defects and lethality. $\boldsymbol{D}, \boldsymbol{E}$, 0verexpression of SPTL-1(C121W) induces mild intestinal polarity phenotype in WT-appearing sptl-1(c363g)/+ adults. White arrow indicates mislocalization of ERM-1 to basolateral membrane. $\boldsymbol{F}$, Localization of Prgef-1::SPTL-1::mCherry and Prgef-1:: SPTL-1(C121W)::mCherry in neuronal cell body and process. $\mathbf{G}, \boldsymbol{H}$, Pan-neuronal overexpression of SPTL-1(C121W), but not SPTL-1, results in VD/DD axon outgrowth defects. $\boldsymbol{I}, \boldsymbol{J}$, Pan-neuronal overexpression of SPTL-1(C121W), but not SPTL-1, causes axon-dendrite identity defects in RIA neurons. Scale bars, $10 \mu \mathrm{m} . \mathbf{C}, \mathbf{D}, \boldsymbol{H}, I$, Quantification of the phenotypes. Three independent experiments were performed with at least 100 worms examined in each. Data are mean \pm SD. Statistical analysis was conducted using the one-way ANOVA, Tukey HSD post hoc test: $F_{(2,6)}=105.2$, $p<0.0001$ for $\boldsymbol{D}$; with two-tailed Student's $t$ test. $\boldsymbol{C}, \boldsymbol{H}, \boldsymbol{I},{ }^{*} p<0.05,{ }^{* * *} p<0.001,{ }^{* * * *} p<0.0001$. 

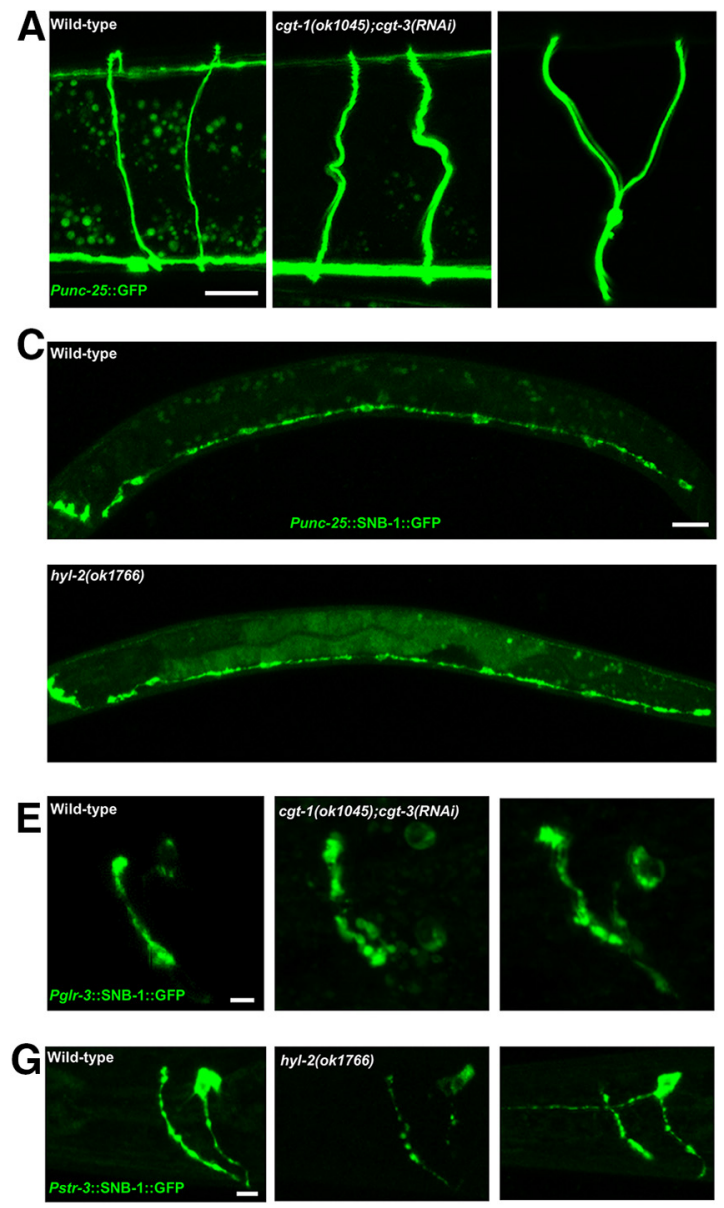
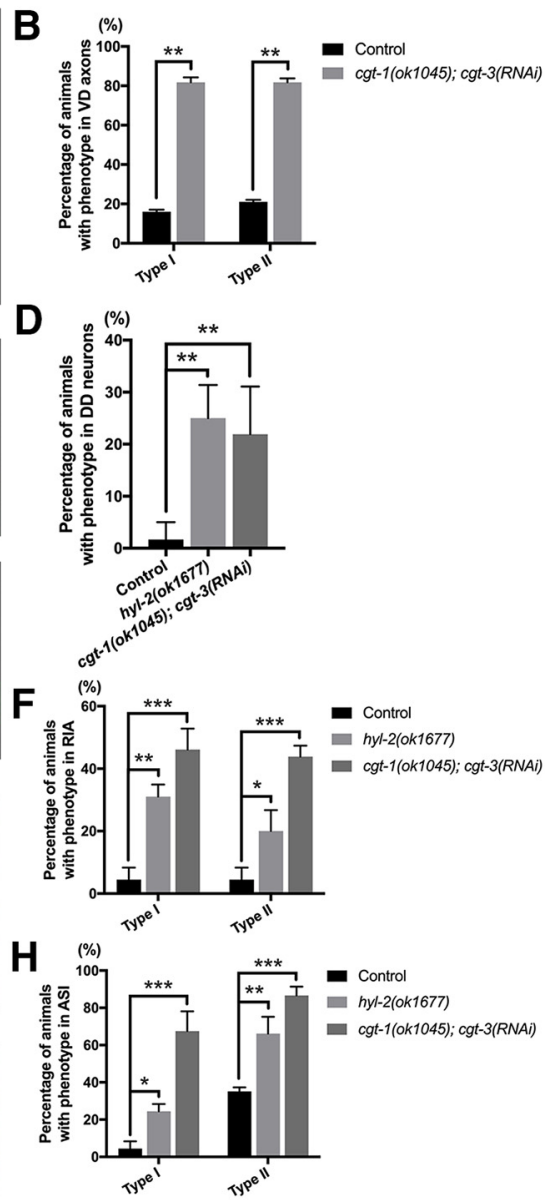

Figure 6. Depleting components of the ceramide synthase or ceramide glucosyltransferase results in phenocopy of the sptl-1(c363g)-, sptl-1(RNAi)-, and SPTL-1(C121W)-induced neuronal morphology and polarity defects. $\boldsymbol{A}, \boldsymbol{B}$, Axon outgrowth defects in L4-stage VD/DD neurons. $\boldsymbol{C}, \boldsymbol{D}$, Defects in axon-dendrite identity of L1 DD neurons. $\boldsymbol{E}$, $\boldsymbol{F}$, Defects in ASI neuronal polarity. $\boldsymbol{G}, \boldsymbol{H}$, Defects in axonal localization of SNB-1 in RIA neurons. $\boldsymbol{B}, \boldsymbol{D}, \boldsymbol{F}, \boldsymbol{H}$, Quantifications for the phenotypes in different backgrounds. Three independent experiments were performed with at least 100 worms examined in each. $\boldsymbol{B}$, Statistical analysis was conducted using two-tailed Student's $t$ test for each type of phenotype in VD axons. Data are mean \pm SD. ${ }^{* *} p<0.01$. D, F, $\boldsymbol{H}, S$ tatistical analysis was conducted using the one-way ANOVA, Tukey HSD post hoc test: $F_{(2,9)}=14.14, p=0.0017(\boldsymbol{D}) ; F_{(2,9)}=14.14, p=0.0017(\boldsymbol{F})$; and $F_{(2,5)}=43.45, p=0.0007(\boldsymbol{H}) .{ }^{*} p<0.05$, ** $p<0.01,{ }^{* * *} p<$ 0.001. Scale bars: $A, C, 10 \mu \mathrm{m} ; E, G, 2 \mu \mathrm{m}$.

$1(c 363 g) /+$ heterozygote adults, demonstrating that the mutated transgene and the germline mutation disrupt the same function (Fig. 5D,E). Together, these data suggest that SPTL-1(C121W) acts in a dominant-negative manner in the $C$. elegans intestine and nervous system.

\section{sptl-1(c363g)-derived epithelial and neuronal phenotypes}

could be caused by a loss of glucosylceramide

Our previous work suggested that SPT and its downstream SLbiosynthetic enzymes regulate epithelial polarity through glycosphingolipids (Zhang et al., 2011). Given that sptl-1(c363g) mutants copy the intestinal polarity phenotype induced by the loss of multiple SL biosynthetic enzymes, including ceramide glucosyltransferases (CGTs) and thus its product glucosylceramide (Zhang et al., 2011), we considered the possibility that lack of glucosylceramide might also be the underlying cause for the $s p t l$ 1(c363g)-derived intestinal and perhaps neuronal phenotype. Although CGT proteins may not be generated in the C. elegans nervous system (Marza et al., 2009; Nomura et al., 2011), cgt-3 RNA, however, is expressed (see NEXTDB: http://nematode.lab. nig.ac.jp); and although glycosphingolipids are not expected to move freely through membranes like their precursors, such as sphinganine (Marza et al., 2009), they might reach the nervous system through different routes. To explore whether the neuro- nal phenotypes of $s p t l-1$ mutants could also be caused by loss of glucosylceramide, the homozygous mutants of several downstream enzymes, including the ceramide synthases $h y l-1$ and $h y l-2$ and the CGTs cgt- 1 and cgt-3, were evaluated for copy of the neuronal defects. hyl-2(ok1766) (RRID:WB-STRAIN:RB1498) homozygous mutants that have reduced but not full loss of ceramide synthase activity displayed neuronal polarity phenotypes in multiple types of neurons similar to that of the progeny of sptl-1(c363g)/+ heterozygous mutants (Fig. 6C-H). We did not detect neuronal defects in cgt-1(ok1045) mutants. This is not unexpected, as cgt-1 is functionally redundant with cgt-3 (Marza et al., 2009; Nomura et al., 2011). We therefore performed cgt-3 RNAi in cgt-1(ok1045) mutants. Progeny of cgt-1(ok1045);cgt3(RNAi) double-mutant parents exhibited L1 arrest with epithelial polarity defects, as well as DD neuron polarity defects (Fig. $6 C, D)$. Mild cgt-3 RNAi initiated at the L1-stage in cgt-1(ok1045) mutants led to the same defects in DD/VD axonal growth and guidance at the L4 stage in the same generation, with even higher penetrance than in sptl-1(c363g)/+ mutant progeny (Fig. 6A-H). Since CGT is the furthest downstream enzyme in this biosynthetic pathway, these findings suggest that lack of glucosylceramide is the common cause for the neuronal phenotypes observed in all these animals with defects in upstream SL biosynthetic enzymes and consequent lack of substrates for glucosylce- 
ramide synthesis. This would include sptl-1(c363g)/+ animals, further suggesting that "loss" of SL products, rather than "gain" of toxic SL byproducts, causes their phenotypes. Transgenic overexpression of CGT in the intestine or nervous system of sptl1(c363g) mutant animals was unable to rescue the epithelial polarity and larval lethality phenotypes (data not shown), consistent with a lack of upstream substrate for glucosylceramide biosynthesis. Collectively, these data suggest that glycosphingolipids not only regulate epithelial, but also neuronal, polarity. They moreover demonstrate that, contrary to current assumptions (Marza et al., 2009), glycosphingolipids, as in most other species, have a critical function in the C. elegans nervous system.

\section{Sphingolipidomic analysis revealed reduced complex SL levels in SPTL-1(C121W) transgenic animals}

To further elucidate the molecular pathogenesis of HSAN1, we performed sphingolipidomic analysis on C. elegans. The C. elegans sphingolipidome is considerably smaller than that of yeast, Drosophila, and mammalian because of the presence of a single C17 sphingoid base (Chitwood et al., 1995). Nonetheless, C. elegans has all functionally distinct sphingoid derivatives, including simple SLs (e.g., sphinganine [d17:0 Sa] and sphingosine [d17:1 So]); N-acyl-derivatives (e.g., ceramide [Cer] and ceramide 1-phosphate [C1P]); and complex SLs (e.g., sphingomyelins [SM] and glucosylceramides [GluCer]), as well as their hydroxylated products (e.g., CerOH, $\mathrm{C} 1 \mathrm{POH}, \mathrm{SMOH}$, and Glu$\mathrm{CerOH})$. The detection of these compounds was optimized individually for chromatography column mode, collision energy, and solvent (X.J. et al., unpublished observations). We also identified atypical DSBs (Fig. 7-1 A, available at https://doi.org/10.1523/ JNEUROSCI.2541-18.2019.f7-1). The identification of the two C. elegans DSBs was verified by monitoring the elution peaks together with the exact mass (Fig. 7-1B-D, available at https:// doi.org/10.1523/JNEUROSCI.2541-18.2019.f7-1).

Among $\sim 170$ individual SL species that we detected, 54 showed significant abundance in WT animals (Fig. 7-2, available at https://doi.org/10.1523/JNEUROSCI.2541-18.2019.f7-2). Complex SLs and ceramides contain a C17 sphinganine with an amide-linked fatty acid moiety varying from 20 to 26 carbon atoms, with $\mathrm{C} 22$ being the most abundant, corroborating previous results (Zhang et al., 2011). We also consistently detected free sphingoid bases, such as d17:0 Sa, d17:1 So, and m17:0 DoSa (Fig. 7-2, available at https://doi.org/10.1523/ JNEUROSCI.2541-18.2019.f7-2), but only at low abundance (less than $10 \%$ of complex SLs).

We then performed SL profiling, focusing on 36 compounds with relatively high abundance, by using the SPTL-1 OE and SPTL-1(C121W) OE transgenic lines. To obtain large homogeneous populations of animals for this quantitative analysis, the transgenes were integrated into the genome. Likely due to lower copy number integration of a transgene integrated SPTL-1(C121W) lines, similar to heterozygote sptl-1(c363g) germline mutants, have no L1 lethality and intestinal polarity defects, and are thus not expected to have dramatic differences in their SL profiles. Despite this, N2, SPTL-1 OE, and SPTL-1(C121W) OE animals could be separated in the primary component analysis score plots (Fig. 7-3A, available at https://doi.org/10.1523/JNEUROSCI. 2541-18.2019.f7-3), suggesting that they harbored measurable differences in SL profiles. This was confirmed on a heat map (Fig. $7 A$ ) where most of the quantified compounds showed differential expression. SPTL-1(C121W) OE animals displayed a $30-$ $40 \%$ decrease in total SL level and $20-60 \%$ decreases for most of complex SL species (SM and GluCer), compared with SPTL-1
OE and WT (N2) animals (Fig. 7B-D; Fig. 7-3, available at https://doi.org/10.1523/JNEUROSCI.2541-18.2019.f7-3). The most striking decrease was observed for GluCer, with levels of all GluCer species decreasing significantly and 7 of 15 compounds with markedly altered VIP scores belonging to the GluCer class (Fig. 7E,F; Fig. 7-3, available at https://doi.org/10.1523/ JNEUROSCI.2541-18.2019.f7-3). In contrast, expected decreases in their precursors ( $\mathrm{Sa}$, So, and Cer) could not be discerned, likely due to the low abundance of sphingoid bases that we measured relative to complex SLs (see above). Nevertheless, we did detect a significant increase in m17:0 DoSa in SPTL-1(C121W) OE animals, as reported in other HSAN1 models (Fig. 7B). Collectively, we find it reasonable to infer the expected reduction in SPTL-1's enzymatic function in SPTL-1(C121W) OE animals from the observed decrease in complex SLs.

Together with our genetic analysis, these sphingolipidomic data are therefore compatible with the notion that the SPTL$1(\mathrm{C} 121 \mathrm{~W})$ mutation acts in a dominant-negative manner reducing SPT activity, as previously suggested (Bejaoui et al., 2002; Gable et al., 2002; Dedov et al., 2004; McCampbell et al., 2005), with a resultant overall decrease in SLs, including glycosphingolipids. Since loss of glycosphingolipids suffices to generate the SPTL-1(C121W) phenotype, it appears less likely, albeit not excluded, that a second independent cause-toxicity of DSB-induces it.

\section{SLs may regulate axon-dendrite identity in neurons through AP3- and kinesin-mediated vesicular trafficking}

In the C. elegans intestine, SLs cooperate with the vesicle coat clathrin and its AP-1 adaptor to maintain epithelial polarity by directing apically destined vesicles with glycosphingolipids-rich membrane microdomains (rafts) toward the apical domain (Shafaq-Zadah et al., 2012; Zhang et al., 2012). In the C. elegans nervous system, APB-3 and UNC-101, components of the clathrin adaptors AP-3 (APB-3) and AP-1 (UNC-101), instruct RIA axon-dendrite polarity by directionally targeting selected transmembrane proteins to axons and dendrites, respectively ( $\mathrm{Li}$ et al., 2016). We therefore next examined whether SLs might maintain polarity in neurons in a similar fashion as in epithelia and introduced the sptl-1(c363g) allele into apb-3(ok429) (RRID:WBSTRAIN:RB662) and unc-101(m1) (RRID:WB-STRAIN:DR1) mutants to assess whether SLs and clathrin adaptors cooperate in regulating RIA axon-dendrite polarity. $a p b$-3(ok429) carries an exonic deletion, resulting in a Val 366-stop, and is predicted to be a null mutation (Hermann et al., 2005), whereas unc-101(m1) harbors a nonsense mutation, reducing, but not abolishing, UNC-101 function (Lee et al., 1994). SNB-1::GFP was mislocalized to the postsynaptic (dendritic) region of RIA neurons in a similar percentage of $a p b-3$ (ok429) L4 larvae and L4 larval progeny of $s p t l-1(c 363 g) /+$ parents (Fig. $8 A$; a population containing $2 / 3$ heterozygote and 1/3 WT animals), although the sptl$1(c 363 g) /+$ progeny showed more obvious mislocalization (data not shown). The penetrance of dendritic mislocalization of SNB-1 in RIA neurons was not significantly increased in sptl1(c363g)/+;apb-3(ok429) double-mutant L4 progeny compared with the corresponding single-mutant populations (Fig. $8 A$ ); the observed increase falls short of an additive effect. Moreover, the lesser severity of the $a p b-3(o k 429)$ null mutant phenotype could not be enhanced by the $s p t l-1(c 363 g)$ allele, suggesting that APB-3 and SPTL-1 products function in the same pathway (data not shown). In agreement with previous reports (Li et al., 2016), unc-101(m1) mutant L4 larvae did not show dendritic SNB-1 mislocalization. Moreover, the unc-101 mutation neither en- 
A

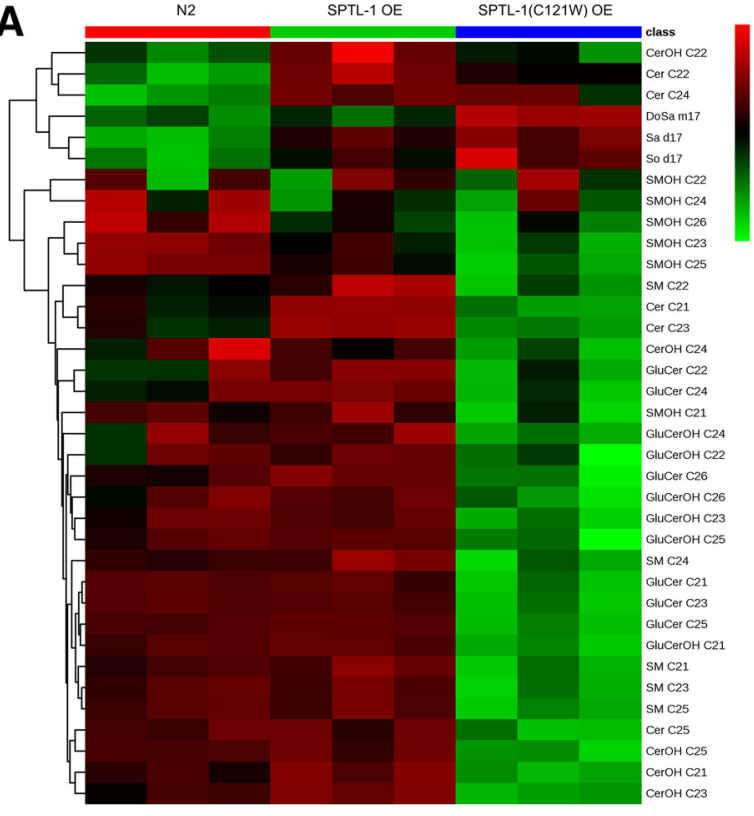

E

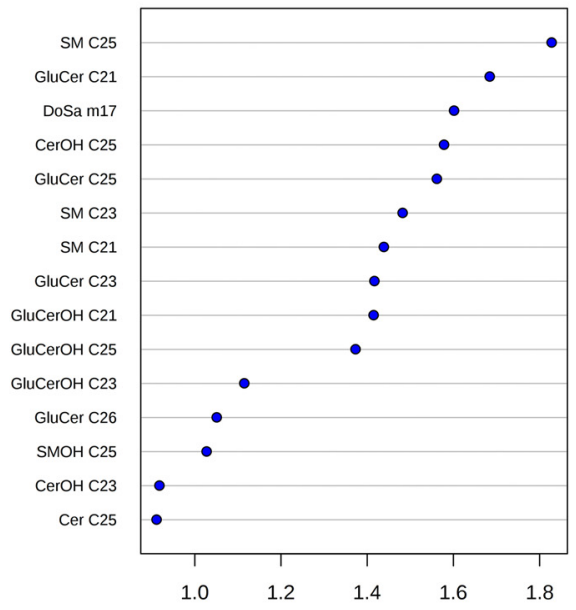

B
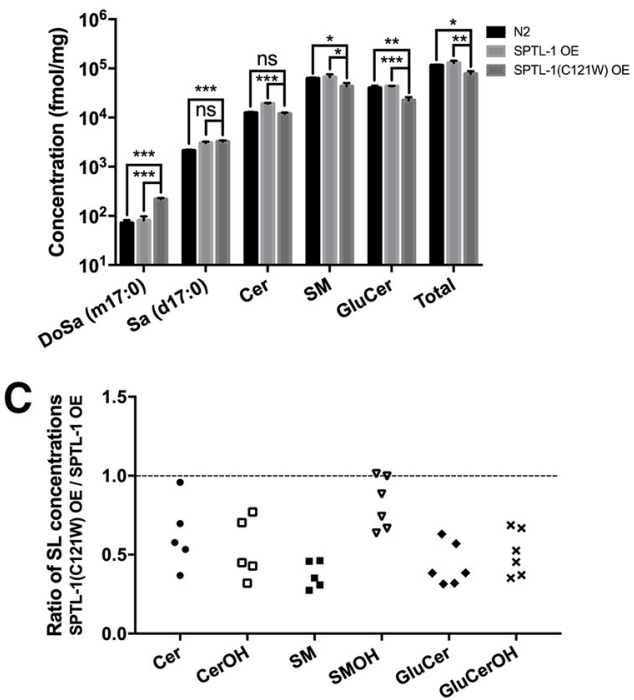

D

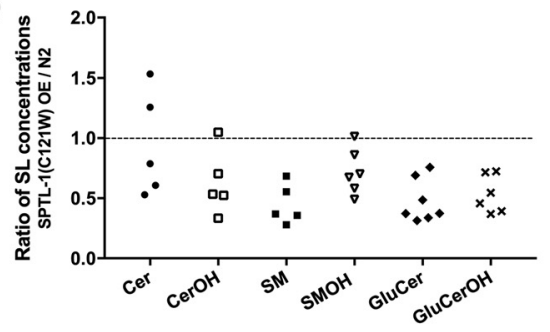

$\mathbf{F}$

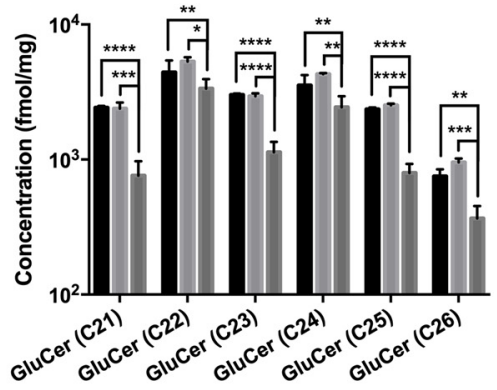

Figure 7. SL profiles are consistent with a dominant-negative effect of SPTL-1(C121W) with remarkably decreased levels of complex SLs. $A$, Hierarchical clustering heat map of the 36 SL compounds quantified in N2, SPTL-1 0E, and SPTL-1(C121W) 0E worms. The color of each section is proportional to the significance of alteration of compounds. Green represents reduction. Red represents increase compared with N2 group. Columns represent individual experiments. Rows represent each quantified compound. B, Quantification of free sphingoid bases (Sa [d17:0] and DoSa [m17:0]) and the sum of each type of SLs of N2, SPTL-1 OE, and SPTL-1(C121W) OE worms. Chemical structures of DSBs in human and C. elegans are shown in Figure 7-1A (available at https://doi.org/10.1523/JNEUROSCI.2541-18.2019.f7-1). Figure 7-1B-D (available at https://doi.org/10.1523/JNEUROSCI.2541-18.2019.f7-1) shows that the identification of the two C. elegans DSBs was verified by monitoring the elution peaks together with the exact mass. C, $D$, Ratio of complex SLs in SPTL-1(C121W) 0E versus SPTL-1 OE animals and in SPTL-1(C121W) 0E versus N2 animals. $E$, Variable importance in projection (VIP) plot: important features (analyzed SL level) identified by PLS-DA in an ascending order of importance. Graph represents relative contribution of SL types to the variance among the N2 animals, SPTL-1 0E animals, and SPTL-1(C121W) 0E animals. Higher value of VIP score indicates greater contribution of the SL type to the group separation. Red represents increased levels. Green represents decreased levels. $F$, Quantification of individual compound with different chain lengths of GluCers in N2, SPTL-1 0E, and SPTL-1(C121W) 0E animals. Graphs of the amounts are shown on a logarithmic scale \pm SD. $n=9$ ( 3 biological replicates $\times 3$ technical replicates). See Figure 7-2 (available at https://doi.org/10.1523/JNEUROSCI.254118.2019.f7-2) for molecular composition of the WT C. elegans sphingolipidome. See Figure 7-3 (available at https://doi.org/10.1523/JNEUROSCI.2541-18.2019.f7-3) for quantification of levels of individual compounds with different chain lengths of other species of SLS. $\boldsymbol{B}, \boldsymbol{F}$, Statistical analysis was conducted using the one-way ANOVA, Tukey HSD post hoctest. $\boldsymbol{B}$, DoSa $(\mathrm{m} 17: 0), F_{(2,5)}=100.5$, $p<0.0001 ; \mathrm{Sa}(\mathrm{d} 17: 0), F_{(2,5)}=38, p=0.0009 ; \mathrm{Cer}_{,} F_{(2,5)}=87.34, p=0.0001 ; \mathrm{SM}_{1} F_{(2,6)}=9.72, p=0.0131 ;$ GluCer, $_{(2,6)}=36.68, p=0.0004 ;$ Total, $F_{(2,5)}=19.28, p=0.0045 . F_{,}$GluCer $(C 21), F_{(2,6)}=67.4, p<0.0001 ; \operatorname{GluCer}(C 22), F_{(2,6)}=5.788, p=0.0398 ; \operatorname{GluCer}(C 23), F_{(2.6)}=139.2, p<0.0001 ; \operatorname{GluCer}(C 24), F_{(2,6)}=10.69, p=0.0105 ; G \operatorname{luCer}\left((25), F_{(2,6)}=294.9, p<\right.$ 0.0001; GluCer (C25), $F_{(2,6)}=39.38, p=0.0004 .{ }^{*} p<0.05,{ }^{* *} p<0.01,{ }^{* * *} p<0.001,{ }^{* * * *} p<0.0001$ and ns (not significant, $p>0.05$ ).

hanced nor suppressed the SNB-1 mislocalization phenotype of sptl-1 $(c 363 g) /+$ progeny. Our results are consistent with the hypothesis that, in RIA neurons, SL-rich vesicles may recruit AP-3, but not AP-1, for axonal vesicle transport.
Vesicular transport in neurons proceeds along microtubule tracks, where molecular motors, such as kinesins and dyneins, move vesicles toward the plus-ends and minus-ends, respectively (Bentley and Banker, 2016). Reducing the function of the C. el- 
A

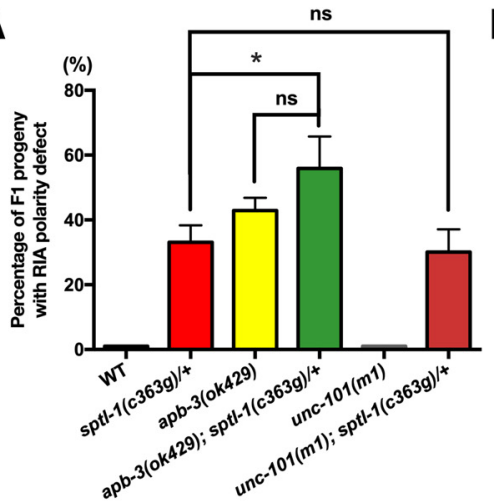

Genotypes of parents

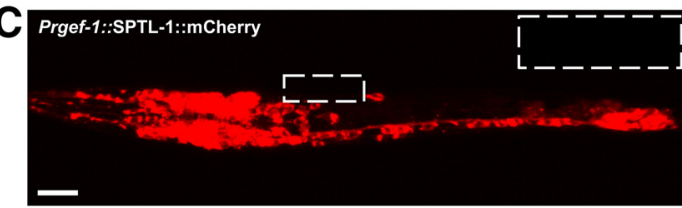

Prgef-1::SPTL-1::mCherry; unc-116(e2310)

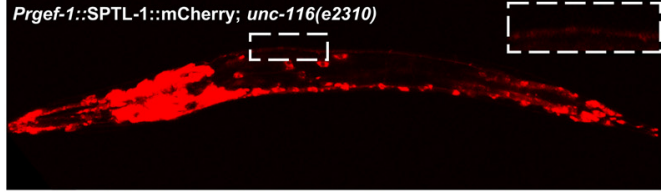

B
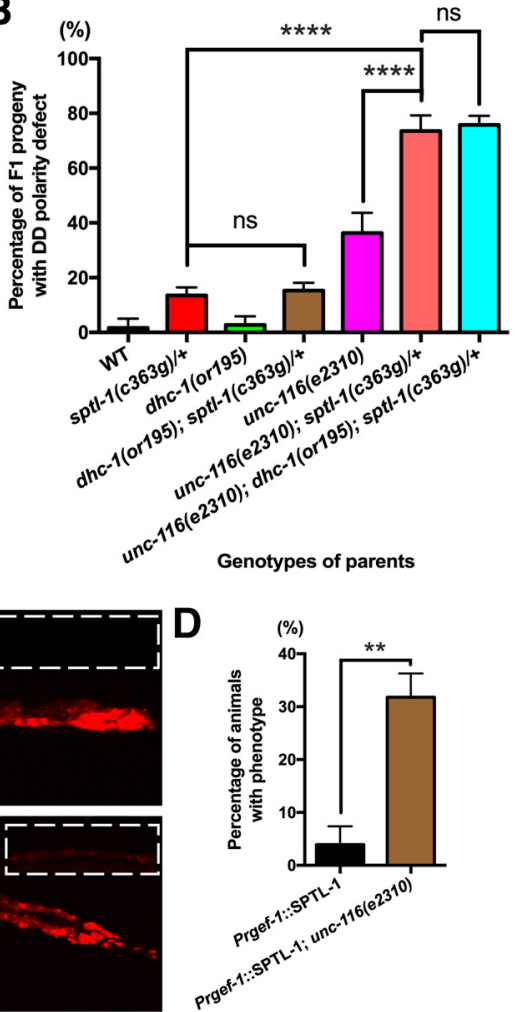

$\mathbf{E}$
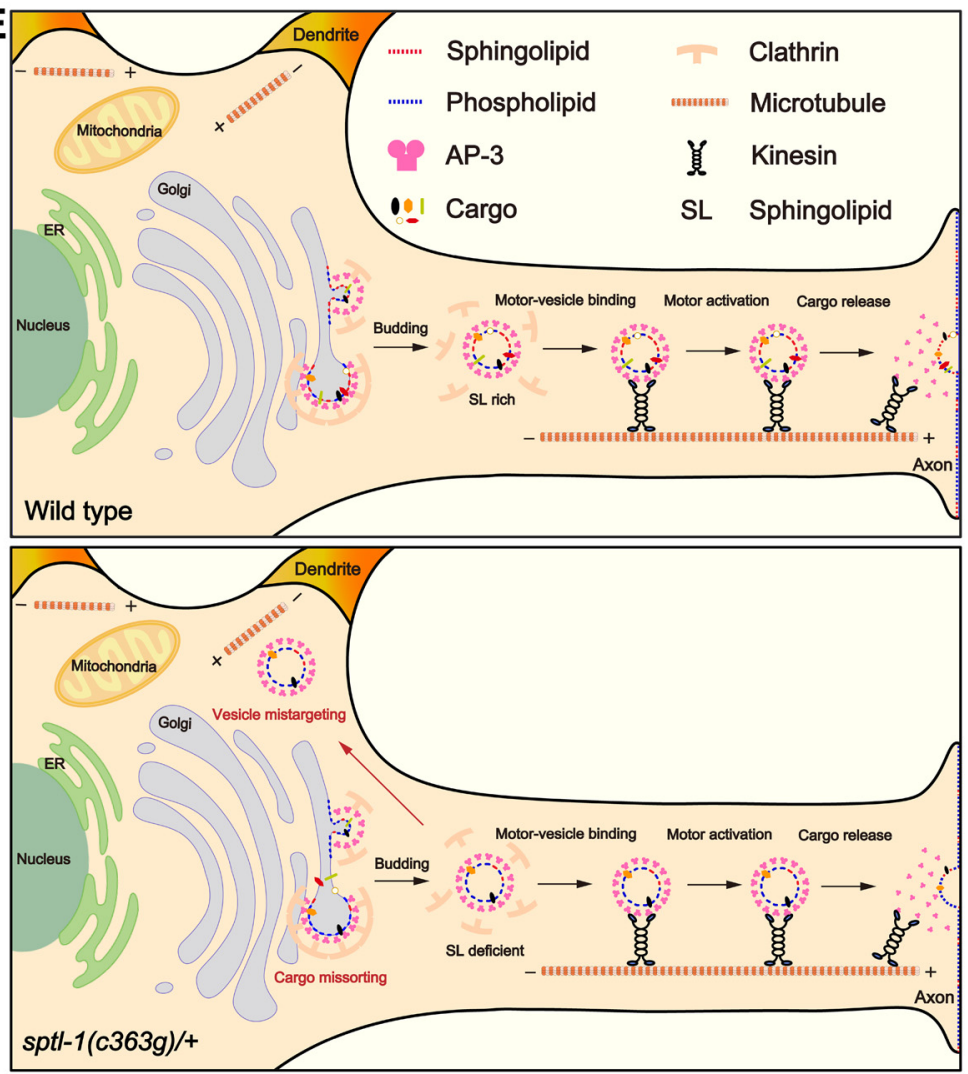

Figure 8. SLs regulate axon-dendrite identity in neurons through AP3- and kinesin-mediated vesicular trafficking. A, Quantification of the SNB-1 mislocalization phenotype in RIA neurons of different strains. $B$, Quantification of the polarity defect in DD neurons of different strains. $n>100$ worms for each strain. $\boldsymbol{A}, \boldsymbol{B}$, Statistical analysis was conducted using the one-way ANOVA, Tukey HSD post hoc test: $F_{(5,10)}=48.69(\boldsymbol{A}), F_{(6,21)}=188.5(\boldsymbol{B}), p<0.0001 .{ }^{*} p<0.05 .^{* *} p<0.01 .{ }^{* * *} p<0.0001$ and ns (not significant, $p>0.05)$. C, D, Ectopic SPTL-1 localization in dorsal processes of presumptive L1 DD neurons in unc-116(e2310) mutants and quantification. Scale bars, $10 \mu \mathrm{m} . n>50$ worms for each strain. Statistical analysis was conducted using two-tailed Student's $t$ test. Data are mean \pm SD. ${ }^{*} p<0.05$. E, A proposed model for the pathogenic mechanism of the SPTL-1(C121W) egans kinesin heavy chain component UNC-116 also results in mislocalization of SNB-1 presynaptic vesicles to the dorsal side of the DD neurons in L1 animals (Byrd et al., 2001). To examine whether SLs might function together with molecular motors in synaptic vesicle localization, we tested genetic interactions between SLs and kinesin or dynein. The unc116(e2310) (RRID:WB-STRAIN:FF41) allele carries a transposon insertion and behaves as a partial loss-of-function mutation (Patel et al., 1993), and a point mutation in dhc-1(or195) (RRID:WBSTRAIN:EU828) reduces the activity of this dynamin heavy chain component to a low, but not null, level (O'Rourke et al., 2007). Consistent with enhancement, unc-116(e2310);sptl-1(c363g)/+ doublemutant L1 progeny (a population containing 50\% sptl-1(c363g)/+ animals), mislocalized more SNB-1::GFP puncta along the length of the dorsal processes of DD neurons than single-mutant populations, and the percentage increase of animals with phenotype in the doublemutant population exceeded the increase expected for an additive effect (Fig. 8B). In contrast, reducing DHC-1 function in $d h c-1$ (or195) mutants neither led to such polarity defects in DD neurons in a WT background nor modified the polarity defect in sptl$1(c 363 g) /+$ progeny. We conclude that the kinesin UNC-116, but not the dynamin DHC-1, may interact with SL-rich vesicles in synaptic vesicle localization.

Certain kinesins bind preferentially to glycosphingolipid-rich membrane lipid microdomains (rafts) (Klopfenstein et al., 2002). To further investigate a possible interaction between kinesin and SLs in neuronal polarity, we examined the localization of SPTL-1::mCherry in unc116 mutants. SPTL-1::mCherry signals, expressed from a pan-neuronal promoter, are mainly concentrated in neuronal cell bodies but are also distributed along some nerve processes (e.g., the ventral nerve cord). unc-116(e2310) neurons showed an SPTL-1::mCherry localization in soma and processes similar to that in WT (Fig. $8 C, D)$. However, before polarity reversion at the L2 stage, when SNB-1 is targeted to

\section{$\leftarrow$}

mutation. In WT neurons, SL-rich microdomains assemble the axonally destined vesicles by recruiting clathrin adaptor AP3 and axonal protein components, which travel along the microtubule with the assistance of the motor kinesin. In SPTL1(C121W) neurons, SL deficiency impairs axonal transport due to either vesicle mistargeting or cargo missorting. 
presynaptic regions at the dorsal side, SPTL-1::mCherry was detected at the dorsal side in unc-116(e2310), but not in WT, L1 larvae (Fig. $8 C, D$ ), similar to what was observed for SNB-1 in sptl-1(c363g)/+ L1 mutant progeny (Fig. 4E).

Complex SL biosynthesis takes place on endomembranes, including Golgi membranes, and the maturation of microdomains on vesicle membranes begins at the Golgi (Lingwood and Simons, 2010). Although not expected to directly reflect the location of lipid rafts on vesicle membranes, the ER-Golgibased SPTL-1 and other lipid-biosynthetic enzymes may be spatially associated with such domains during cellular polarization (H.Z. and V.G., unpublished observations). We thus interpret these results as indication for a possible direct involvement of UNC-116 in the axonal targeting of SL- rich endomembrane microdomains.

\section{Discussion}

HSAN1 is an inherited disease that has been investigated in a range of cell lines and animal models; however, none of these experimental settings created the exact genetic condition of human HSAN1 (Gable et al., 2002; McCampbell et al., 2005; Oswald et al., 2015). By using CRSPR-mediated gene editing, we here generated C. elegans sptl-1 $(c 363 g) /+$ mutants that are genetically equivalent to HSAN1 patients carrying the C133W mutation in SPTLC1, the first rate-limiting SL biosynthetic enzyme. sptl$1(c 363 g) /+$ mutant worms displayed several neuronal phenotypes, including sensory dysfunction, that resemble HSAN1 associated motor and sensory defects, suggesting that $C$. elegans is a suitable model for the disease. The pathogenesis of HSAN1 remains unclear, with current hypotheses favoring the accumulation of a toxic byproduct (DSB) as the underlying cause of the disease (see Introduction). Taking advantage of the genetic versatility of $C$. elegans, we here provide evidence for a dominantnegative loss-of-function effect of the SPTL-1(C121W) mutation that results in loss of its downstream SL products, specifically glycosphingolipids, suggesting an alternate mechanism for the mutations' cellular effects. Our analysis further revealed that these effects might be mediated by impaired SL-dependent polarized vesicular trafficking, suggesting that defects in polarity maintenance could be the underlying cause of neuronal degeneration and dysfunction in HSAN1. Our findings thus propose a novel pathogenetic mechanism for this disease with immediate implications for therapeutic strategies.

\section{The conserved function of the SPTL-1 C121 residue and its pathogenetic effect}

A major finding of this study is that $\mathrm{C} 121$ is an important residue for SPTL-1 function, not only in SL biosynthesis but also in maintaining epithelial and neuronal polarity. Strikingly, a single point mutation of sptl-1(c363g) sufficed to phenocopy the wellcharacterized sptl-1 loss-of-function epithelial polarity phenotype and cause larval lethality, strongly implicating the loss of SPT activity and the subsequent loss of SLs in $s p t l-1(c 363 g)$-induced perturbations. The critical steps of our genetic analysis all supported this notion: (1) copy of all sptl-1(c363g) phenotypes by knockdown of sptl-1; (2) recessive inheritance pattern of the sptl1(c363g) epithelial and lethal phenotypes; (3) rescue of these phenotypes by transgenic copies of sptl-1; and (4) copy of the epithelial (Zhang et al., 2011) and neuronal phenotypes by loss-of-function defects in several downstream SL-biosynthetic enzymes. We therefore interpret the dominant pattern of the SPTL$1(\mathrm{C} 121 \mathrm{~W})$ mutant transgene as a dominant-negative loss-of- function effect resulting in loss of enzyme activity, rather than a gain-of-function effect. This assumption is supported by this transgene's ability to induce a synthetic epithelial polarity defect in otherwise WT-appearing c363g/+ heterozygote animals, and is corroborated by reduced complex SL levels measured in these transgenic strains. The interpretation fits well with structural data from Sphingomonas paucimobilis on the highly conserved SPT that must heterodimerize in humans for function: the model predicts that the C121 corresponding C133 residue of SPTLC1 lies at the dimer interface close to SPTLC2's residue involved in substrate binding, such that C133 mutations directly affect the interaction of C133's side chain with SPTLC2's key residue Thr378, thereby disrupting the active site of the dimer (Raman et al., 2009). Depletion of a heterodimer required for function represents a classical scenario for a dominant-negative loss-of-function effect (Wilkie, 1994). We therefore also suggest that the dominant inheritance pattern of the neuronal phenotype (present in $c 363 g /+$ heterozygote animals) reflects a sensitivity of the nervous system to impaired SL biosynthesis and thus, haploinsufficiency of the locus for the nervous system. A highly polarized nervous system could, for instance, be specifically sensitive for polarity defects, here suggested to cause the neuropathology (see below). Nevertheless, the increase in deoxy-SLs detected in these transgenic animals does not preclude the possibility that DSBs, shown to have toxicity in the C. elegans intestine (Hannich et al., 2017), may also have a toxic effect on the C. elegans nervous system.

\section{Glycosphingolipids maintain neuronal polarity and their loss may cause HSAN1-like neuropathology by impairing axonal trafficking}

The strongest evidence for a loss-of-function effect of splt$1(c 363 g)$ mutation comes from the exact copy of its epithelial and neuronal polarity phenotypes by the knockdown of SPT and multiple downstream SL biosynthetic enzymes that all require SPT products as their direct or indirect substrates (Zhang et al., 2011). Phenocopy by the loss of the furthest downstream enzyme CGT thus strongly suggests that it is the loss of glucosylceramide that causes all defects, although we cannot formally rule out the loss of any other intermediate as the underlying cause. Glucosylceramide and other complex glycosphingolipids (e.g., gangliosides) have long been recognized for their conserved roles in neural development (Schengrund, 2015). Glycosphingolipids' role in polarity established in epithelia is linked to polarized trafficking via apical cargo sorting on glycosphingolipid- and cholesterol-rich membrane microdomains (rafts), and has also been implicated in neuronal development (Ledesma et al., 1998; Rodriguez-Boulan et al., 2005). Neurons have striking cellular divisions as axons and dendrites and therefore require particularly efficient mechanisms for polarized transport to generate and maintain these distinct domains, consistent with high sensitivity to defects in polarized trafficking. Glycosphingolipids may thus also function as vesicular sorting signals in neurons, where SLrich vesicles could serve as carriers for axonal components. The sptl-1(c363g) mutation may impair axonal trafficking by inhibiting SL-, and thereby GluCer- biosynthesis, resulting in cargo missorting or vesicle mistargeting during axonal transport (Fig. 8E). The finding that SPTL-1 functionally interacts with APB-3 and UNC-116 in polarized SNB-1 localization in multiple types of $C$. elegans neurons is consistent with such a hypothesis and could suggest that SL-rich vesicles support microtubule-based vesicular transport of axonal components with the aid of kinesin and through recruiting the clathrin adaptor AP-3 (Fig. 8E).

Defects in SL-dependent directional trafficking could also explain characteristics of HSAN1 neuropathology. Interestingly, 
other disease-causing HSAN1 genes, such as ATL1/atlastin-1, a large dynamin-related GTPase, and RAB7, a small Rab GTPase, also function in vesicular trafficking. ATL1 resides at ER membranes (Orso et al., 2009), and loss of ATL-1 function alters ER structure in motor axons and nerve terminals and affects neuronal function through bone morphogenetic protein trafficking and signaling in both Drosophila and zebrafish (Fassier et al., 2010; Summerville et al., 2016). Interestingly, ATL1 depletion in developing rat cortical neurons inhibited axonal elongation, and overexpression of a mutant form (devoid of GTPase activity) of ATL1 during murine development resulted in dendrite morphogenesis defects (Gao et al., 2013; Shih and Hsueh, 2016), probably due to the disruption of ATL-1-mediated membrane dynamics in the neuronal growth cone. RAB7, mutated in Charcot-MarieTooth disease Type 2B (a subtype of HSAN1), plays important roles in vesicular transport to late endocytic compartments and in trafficking and degradation of several signaling receptors (Gao et al., 2013). For instance, Rab7 CMT2B mutants showed impaired growth factor receptor transport and endosomal and nuclear signaling, thereby interfering with normal axon outgrowth and peripheral innervation (BasuRay et al., 2013; Zhang et al., 2013). Defects in axonal transport of Rab7-positive vesicles were observed in Drosophila and zebrafish sensory neurons as well as in different neuronal cells expressing the CMT2B RAB7 mutation (Zhang et al., 2013; Janssens et al., 2014; Ponomareva et al., 2016). The key role of vesicular trafficking in the pathogenesis of this family of HSAN1-related neuropathies thus indirectly supports our hypothesis that the observed HSAN1 neuropathy is caused by impaired vesicular trafficking, in this case, however, as a direct consequence of a glycosphingolipid-dependent defect in directional transport that perturbs the maintenance of neuronal polarity.

\section{Therapeutic implications for HSAN1 and other diseases of SL metabolism}

Perturbations in SL metabolism have been implicated in the pathogenesis of many diseases, including diabetes, cancer, neurodegenerative diseases, and cardiovascular diseases (Meikle and Summers, 2017; Hannun and Obeid, 2018). Moreover, sphingolipidoses represent a group of devastating diseases that are the direct consequence of specific defects in SL metabolism (Xu et al., 2010). Yet, little is known about the cell biological functions that are affected by SL changes in either group of diseases. Even less is known about SLs' roles in disease pathogenesis and SLs' potential to serve as drugs or treatment targets. The term "storage disease," applied to many diseases of lipid metabolism, reflects the current assumption that disease symptoms are mainly caused by the accumulation of toxic intermediates, with therapies focused on preventing their accumulation and alleviating their toxicity. Our findings suggest that the alternative treatment approach, resupplementation of the missing lipid products, also deserves serious consideration. In particular, the option of oral supplementation, open to lipids (as opposed to proteins), would facilitate therapeutic intervention and increase the hope for specific therapies. Supplementation of exogenous sphingoid bases had impressive rescuing effect in Drosophila and C. elegans with SPT deficiency (Adachi-Yamada et al., 1999; Zhu et al., 2013), suggesting that HSAN1 may also be amenable to supplementation of SPT products. The range of downstream products of this firststep SL-biosynthetic enzyme is broad, ranging from sphinganine to GluCer, the latter directly targeting the proposed neuronal polarity defect. However, little is known about the uptake of complex SLs into cells in general and into nerve cells in particular.
Lipid biosynthetic pathways are circular, and compensatory upregulation or downregulation of different pathway arms to maintain lipid homeostasis has been characterized (Köberlin et al., 2015), broadening the range of potential treatment options. Cross regulation between different membrane lipids, such as SLs, phospholipids, and glycerolipids (Nohturfft and Zhang, 2009), further expands this range. If, as suggested here, defects in SL-rich membrane microdomains critically affect HSAN1 pathogenesis, additional approaches may be possible. The rescue of C. elegans larval lethality, induced by defects in glycosphingolipid biosynthesis, via cholesterol supplementation, for instance, not only demonstrates the plasticity of the system but could also suggest an alternate cause for the rescue mechanism: the support of membrane lipid rafts that are rich in both glycosphingolipids and cholesterol (Boland et al., 2017). Our newly established C. elegans model of HSAN1 is well suited to perform forward screens to search the genome for endogenous molecules that may alleviate the neuropathology and to mine small-molecule libraries to identify new exogenous compounds for therapeutic intervention.

\section{References}

Adachi-Yamada T, Gotoh T, Sugimura I, Tateno M, Nishida Y, Onuki T, Date H (1999) De novo synthesis of sphingolipids is required for cell survival by down-regulating c-jun N-terminal kinase in Drosophila imaginal discs. Mol Cell Biol 19:7276-7286.

Arribere JA, Bell RT, Fu BX, Artiles KL, Hartman PS, Fire AZ (2014) Efficient marker-free recovery of custom genetic modifications with CRISPR/ Cas9 in Caenorhabditis elegans. Genetics 198:837-846.

Bargmann CI, Hartwieg E, Horvitz HR (1993) Odorant-selective genes and neurons mediate olfaction in C. elegans. Cell 74:515-527.

BasuRay S, Mukherjee S, Romero EG, Seaman MN, Wandinger-Ness A (2013) Rab7 mutants associated with Charcot-Marie-Tooth disease cause delayed growth factor receptor transport and altered endosomal and nuclear signaling. J Biol Chem 288:1135-1149.

Bejaoui K, Wu C, Scheffler MD, Haan G, Ashby P, Wu L, de Jong P, Brown RH Jr (2001) SPTLC1 is mutated in hereditary sensory neuropathy, type 1. Nat Genet 27:261-262.

Bejaoui K, Uchida Y, Yasuda S, Ho M, Nishijima M, Brown RH Jr, Holleran WM, Hanada K (2002) Hereditary sensory neuropathy type 1 mutations confer dominant-negative effects on serine palmitoyltransferase, critical for sphingolipid synthesis. J Clin Invest 110:1301-1308.

Bentley M, Banker G (2016) The cellular mechanisms that maintain neuronal polarity. Nat Rev Neurosci 17:611-622.

Bertea M, Rütti MF, Othman A, Marti-Jaun J, Hersberger M, von Eckardstein A, Hornemann T (2010) Deoxysphingoid bases as plasma markers in diabetes mellitus. Lipids Health Dis 9:84.

Boland S, Schmidt U, Zagoriy V, Sampaio JL, Fritsche RF, Czerwonka R, Lübken T, Reimann J, Penkov S, Knölker HJ, Kurzchalia TV (2017) Phosphorylated glycosphingolipids essential for cholesterol mobilization in Caenorhabditis elegans. Nat Chem Biol 13:647-654.

Brenner S (1974) The genetics of Caenorhabditis elegans. Genetics 77:71-94. Byrd DT, Kawasaki M, Walcoff M, Hisamoto N, Matsumoto K, Jin Y (2001) UNC-16, a JNK-signaling scaffold protein, regulates vesicle transport in C. elegans. Neuron 32:787-800.

Chitwood DJ, Lusby WR, Thompson MJ, Kochansky JP, Howarth OW (1995) The glycosylceramides of the nematode Caenorhabditis elegans contain an unusual, branched-chain sphingoid base. Lipids 30:567-573.

Chong J, Soufan O, Li C, Caraus I, Li S, Bourque G, Wishart DS, Xia J (2018) MetaboAnalyst 4.0: towards more transparent and integrative metabolomics analysis. Nucleic Acids Res 46:W486-W494.

Crump JG, Zhen M, Jin Y, Bargmann CI (2001) The SAD-1 kinase regulates presynaptic vesicle clustering and axon termination. Neuron 29:115-129.

Dawkins JL, Hulme DJ, Brahmbhatt SB, Auer-Grumbach M, Nicholson GA (2001) Mutations in SPTLC1, encoding serine palmitoyltransferase, long chain base subunit-1, cause hereditary sensory neuropathy type I. Nat Genet 27:309-312.

Dedov VN, Dedova IV, Merrill AH Jr, Nicholson GA (2004) Activity of partially inhibited serine palmitoyltransferase is sufficient for normal sphingolipid metabolism and viability of HSN1 patient cells. Biochim Biophys Acta 1688:168-175. 
Eichler FS, Hornemann T, McCampbell A, Kuljis D, Penno A, Vardeh D, Tamrazian E, Garofalo K, Lee HJ, Kini L, Selig M, Frosch M, Gable K, von Eckardstein A, Woolf CJ, Guan G, Harmon JM, Dunn TM, Brown RH Jr (2009) Overexpression of the wild-type SPT1 subunit lowers desoxysphingolipid levels and rescues the phenotype of HSAN1. J Neurosci 29:14646-14651.

Fassier C, Hutt JA, Scholpp S, Lumsden A, Giros B, Nothias F, SchneiderMaunoury S, Houart C, Hazan J (2010) Zebrafish atlastin controls motility and spinal motor axon architecture via inhibition of the BMP pathway. Nat Neurosci 13:1380-1387.

Gable K, Han G, Monaghan E, Bacikova D, Natarajan M, Williams R, Dunn TM (2002) Mutations in the yeast LCB1 and LCB2 genes, including those corresponding to the hereditary sensory neuropathy type I mutations, dominantly inactivate serine palmitoyltransferase. J Biol Chem 277:10194-10200.

Gao Y, Jiang T, Qu C, Tao H, Cao H, Zhao Y, Wang Y, Qu J, Chen JG (2013) Atlastin-1 regulates dendritic morphogenesis in mouse cerebral cortex. Neurosci Res 77:137-142.

Garofalo K, Penno A, Schmidt BP, Lee HJ, Frosch MP, von Eckardstein A, Brown RH, Hornemann T, Eichler FS (2011) Oral L-serine supplementation reduces production of neurotoxic deoxysphingolipids in mice and humans with hereditary sensory autonomic neuropathy type 1 . J Clin Invest 121:4735-4745.

Hannich JT, Mellal D, Feng S, Zumbuehl A, Riezman H (2017) Structure and conserved function of iso-branched sphingoid bases from the nematode Caenorhabditis elegans. Chem Sci 8:3676-3686.

Hannun YA, Obeid LM (2018) Sphingolipids and their metabolism in physiology and disease. Nat Rev Mol Cell Biol 19:175-191.

Hermann GJ, Schroeder LK, Hieb CA, Kershner AM, Rabbitts BM, Fonarev P, Grant BD, Priess JR (2005) Genetic analysis of lysosomal trafficking in Caenorhabditis elegans. Mol Biol Cell 16:3273-3288.

Hobert O (2002) PCR fusion-based approach to create reporter gene constructs for expression analysis in transgenic C. elegans. Biotechniques 32: $728-730$.

Huang X, Cheng HJ, Tessier-Lavigne M, Jin Y (2002) MAX-1, a novel PH/ MyTH4/FERM domain cytoplasmic protein implicated in netrinmediated axon repulsion. Neuron 34:563-576.

Janssens K, Goethals S, Atkinson D, Ermanoska B, Fransen E, Jordanova A, Auer-Grumbach M, Asselbergh B, Timmerman V (2014) Human Rab7 mutation mimics features of Charcot-Marie-Tooth neuropathy type $2 \mathrm{~B}$ in Drosophila. Neurobiol Dis 65:211-219.

Jin X, Pokala N, Bargmann CI (2016) Distinct circuits for the formation and retrieval of an imprinted olfactory memory. Cell 164:632-643.

Klopfenstein DR, Tomishige M, Stuurman N, Vale RD (2002) Role of phosphatidylinositol $(4,5)$ bisphosphate organization in membrane transport by the Unc104 kinesin motor. Cell 109:347-358.

Köberlin MS, Snijder B, Heinz LX, Baumann CL, Fauster A, Vladimer GI, Gavin AC, Superti-Furga G (2015) A conserved circular network of coregulated lipids modulates innate immune responses. Cell 162:170-183.

Kuervers LM, Jones CL, O'Neil NJ, Baillie DL (2003) The sterol modifying enzyme LET-767 is essential for growth, reproduction and development in Caenorhabditis elegans. Mol Genet Genomics 270:121-131.

Ledesma MD, Simons K, Dotti CG (1998) Neuronal polarity: essential role of protein-lipid complexes in axonal sorting. Proc Natl Acad Sci U S A 95:3966-3971.

Lee J, Jongeward GD, Sternberg PW (1994) unc-101, a gene required for many aspects of Caenorhabditis elegans development and behavior, encodes a clathrin-associated protein. Genes Dev 8:60-73.

Li P, Merrill SA, Jorgensen EM, Shen K (2016) Two clathrin adaptor protein complexes instruct axon-dendrite polarity. Neuron 90:564-580.

Lin CH, Tomioka M, Pereira S, Sellings L, Iino Y, van der Kooy D (2010) Insulin signaling plays a dual role in Caenorhabditis elegans memory acquisition and memory retrieval. J Neurosci 30:8001-8011.

Lingwood D, Simons K (2010) Lipid rafts as a membrane-organizing principle. Science 327:46-50.

Marza E, Simonsen KT, Faergeman NJ, Lesa GM (2009) Expression of ceramide glucosyltransferases, which are essential for glycosphingolipid synthesis, is only required in a small subset of C. elegans cells. J Cell Sci 122:822-833.

McCampbell A, Truong D, Broom DC, Allchorne A, Gable K, Cutler RG, Mattson MP, Woolf CJ, Frosch MP, Harmon JM, Dunn TM, Brown RH Jr (2005) Mutant SPTLC1 dominantly inhibits serine palmitoyltransferase activity in vivo and confers an age-dependent neuropathy. Hum $\mathrm{Mol}$ Genet 14:3507-3521.

Meikle PJ, Summers SA (2017) Sphingolipids and phospholipids in insulin resistance and related metabolic disorders. Nat Rev Endocrinol 13:79-91.

Mello CC, Kramer JM, Stinchcomb D, Ambros V (1991) Efficient gene transfer in C. elegans: extrachromosomal maintenance and integration of transforming sequences. EMBO J 10:3959-3970.

Merrill AH, Sandhoff K (2002) Sphingolipids: metabolism and cell signaling. In: New comprehensive biochemistry, pp 373-407. Amsterdam: Elsevier.

Nohturfft A, Zhang SC (2009) Coordination of lipid metabolism in membrane biogenesis. Annu Rev Cell Dev Biol 25:539-566.

Nomura KH, Murata D, Hayashi Y, Dejima K, Mizuguchi S, Kage-Nakadai E, Gengyo-Ando K, Mitani S, Hirabayashi Y, Ito M, Nomura K (2011) Ceramide glucosyltransferase of the nematode Caenorhabditis elegans is involved in oocyte formation and in early embryonic cell division. Glycobiology 21:834-848.

Nuttley WM, Atkinson-Leadbeater KP, Van Der Kooy D (2002) Serotonin mediates food-odor associative learning in the nematode Caenorhabditis elegans. Proc Natl Acad Sci U S A 99:12449-12454.

O’Rourke SM, Dorfman MD, Carter JC, Bowerman B (2007) Dynein modifiers in C. elegans: light chains suppress conditional heavy chain mutants. PLoS Genet 3:e128.

Orso G, Pendin D, Liu S, Tosetto J, Moss TJ, Faust JE, Micaroni M, Egorova A, Martinuzzi A, McNew JA, Daga A (2009) Homotypic fusion of ER membranes requires the dynamin-like GTPase atlastin. Nature 460: 978-983.

Oswald MC, West RJ, Lloyd-Evans E, Sweeney ST (2015) Identification of dietary alanine toxicity and trafficking dysfunction in a Drosophila model of hereditary sensory and autonomic neuropathy type 1 . Hum Mol Genet 24:6899-6909.

Patel N, Thierry-Mieg D, Mancillas JR (1993) Cloning by insertional mutagenesis of a cDNA encoding Caenorhabditis elegans kinesin heavy chain. Proc Natl Acad Sci U S A 90:9181-9185.

Penno A, Reilly MM, Houlden H, Laurá M, Rentsch K, Niederkofler V, Stoeckli ET, Nicholson G, Eichler F, Brown RH Jr, von Eckardstein A, Hornemann T (2010) Hereditary sensory neuropathy type 1 is caused by the accumulation of two neurotoxic sphingolipids. J Biol Chem 285: $11178-11187$.

Ponomareva OY, Eliceiri KW, Halloran MC (2016) Charcot-Marie-Tooth $2 \mathrm{~b}$ associated Rab7 mutations cause axon growth and guidance defects during vertebrate sensory neuron development. Neural Dev 11:2.

Raman MC, Johnson KA, Yard BA, Lowther J, Carter LG, Naismith JH, Campopiano DJ (2009) The external aldimine form of serine palmitoyltransferase: structural, kinetic, and spectroscopic analysis of the wild-type enzyme and HSAN1 mutant mimics. J Biol Chem 284:17328-17339.

Rodriguez-Boulan E, Kreitzer G, Müsch A (2005) Organization of vesicular trafficking in epithelia. Nat Rev Mol Cell Biol 6:233-247.

Rotthier A, Baets J, De Vriendt E, Jacobs A, Auer-Grumbach M, Lévy N, Bonello-Palot N, Kilic SS, Weis J, Nascimento A, Swinkels M, Kruyt MC, Jordanova A, De Jonghe P, Timmerman V (2009) Genes for hereditary sensory and autonomic neuropathies: a genotype-phenotype correlation. Brain 132:2699-2711.

Rotthier A, Auer-Grumbach M, Janssens K, Baets J, Penno A, Almeida-Souza L, Van Hoof K, Jacobs A, De Vriendt E, Schlotter-Weigel B, Löscher W, Vondrácek P, Seeman P, De Jonghe P, Van Dijck P, Jordanova A, Hornemann T, Timmerman V (2010) Mutations in the SPTLC2 subunit of serine palmitoyltransferase cause hereditary sensory and autonomic neuropathy type I. Am J Hum Genet 87:513-522.

Rotthier A, Penno A, Rautenstrauss B, Auer-Grumbach M, Stettner GM, Asselbergh B, Van Hoof K, Sticht H, Lévy N, Timmerman V, Hornemann T, Janssens K (2011) Characterization of two mutations in the SPTLC1 subunit of serine palmitoyltransferase associated with hereditary sensory and autonomic neuropathy type I. Hum Mutat 32:E2211-E2225.

Rotthier A, Baets J, Timmerman V, Janssens K (2012) Mechanisms of disease in hereditary sensory and autonomic neuropathies. Nat Rev Neurol 8:73-85.

Schengrund CL (2015) Gangliosides: glycosphingolipids essential for normal neural development and function. Trends Biochem Sci 40:397-406.

Shafaq-Zadah M, Brocard L, Solari F, Michaux G (2012) AP-1 is required for the maintenance of apico-basal polarity in the C. elegans intestine. Development 139:2061-2070. 
Shih YT, Hsueh YP (2016) VCP and ATL1 regulate endoplasmic reticulum and protein synthesis for dendritic spine formation. Nat Commun 7:11020.

Sullards MC, Wang E, Peng Q, Merrill AH Jr (2003) Metabolomic profiling of sphingolipids in human glioma cell lines by liquid chromatography tandem mass spectrometry. Cell Mol Biol 49:789-797.

Summerville JB, Faust JF, Fan E, Pendin D, Daga A, Formella J, Stern M, McNew JA (2016) The effects of ER morphology on synaptic structure and function in Drosophila melanogaster. J Cell Sci 129:1635-1648.

Tanizawa Y, Kuhara A, Inada H, Kodama E, Mizuno T, Mori I (2006) Inositol monophosphatase regulates localization of synaptic components and behavior in the mature nervous system of C. elegans. Genes Dev 20:3296-3310.

Teulière J, Gally C, Garriga G, Labouesse M, Georges-Labouesse E (2011) MIG-15 and ERM-1 promote growth cone directional migration in parallel to UNC-116 and WVE-1. Development 138:4475-4485.

White JG, Southgate E, Thomson JN, Brenner S (1986) The structure of the nervous system of the nematode Caenorhabditis elegans. Philos Trans R Soc Lond B Biol Sci 314:1-340.

White JG, Albertson DG, Anness MA (1978) Connectivity changes in a class of motoneurone during the development of a nematode. Nature 271: $764-766$.

Wilkie AO (1994) The molecular basis of genetic dominance. J Med Genet 31:89-98.

Xu YH, Barnes S, Sun Y, Grabowski GA (2010) Multi-system disorders of glycosphingolipid and ganglioside metabolism. J Lipid Res 51:1643-1675

Zhang H, Abraham N, Khan LA, Hall DH, Fleming JT, Göbel V (2011) Apicobasal domain identities of expanding tubular membranes depend on glycosphingolipid biosynthesis. Nat Cell Biol 13:1189-1201.

Zhang H, Kim A, Abraham N, Khan LA, Hall DH, Fleming JT, Gobel V (2012) Clathrin and AP-1 regulate apical polarity and lumen formation during C. elegans tubulogenesis. Development 139:2071-2083.

Zhang H, Abraham N, Khan LA, Gobel V (2015) RNAi-based biosynthetic pathway screens to identify in vivo functions of non-nucleic acid-based metabolites such as lipids. Nat Protoc 10:681-700.

Zhang K, Fishel Ben Kenan R, Osakada Y, Xu W, Sinit RS, Chen L, Zhao X, Chen JY, Cui B, Wu C (2013) Defective axonal transport of Rab7 GTPase results in dysregulated trophic signaling. J Neurosci 33:7451-7462.

Zhang Y, Lu H, Bargmann CI (2005) Pathogenic bacteria induce aversive olfactory learning in Caenorhabditis elegans. Nature 438:179-184.

Zhu H, Shen H, Sewell AK, Kniazeva M, Han M (2013) A novel sphingolipid-TORC1 pathway critically promotes postembryonic development in Caenorhabditis elegans. Elife 2:e00429.

Zitomer NC, Mitchell T, Voss KA, Bondy GS, Pruett ST, Garnier-Amblard EC, Liebeskind LS, Park H, Wang E, Sullards MC, Merrill AH Jr, Riley RT (2009) Ceramide synthase inhibition by fumonisin B1 causes accumulation of 1-deoxysphinganine: a novel category of bioactive 1-deoxysphingoid bases and 1-deoxydihydroceramides biosynthesized by mammalian cell lines and animals. J Biol Chem 284:4786-4795. 